\title{
Dietary suppression of MHC-II expression in intestinal stem cells enhances intestinal tumorigenesis
}

Semir Beyaz 1, 2, 3, 17 , Jatin Roper ${ }^{3,4,16}$, Khristian E. Bauer-Rowe ${ }^{3,16}$, Michael E. Xifaras ${ }^{1,}$ 3, 16, Ilgin Ergin', Lenka Dohnalova ${ }^{5}$, Moshe Biton ${ }^{6,7}$, Karthik Shekar ${ }^{6}$, Haiwei Mou ${ }^{1}$, Onur Eskiocak ${ }^{1}$, Deniz M. Özata ${ }^{8}$, Katherine Papciak ${ }^{1}$, Charlie Chung ${ }^{1}$, Mohammed Almeqdadi $^{3}$, Miriam Fein ${ }^{1}$, Eider Valle-Encinas ${ }^{3}$, Aysegul Erdemir ${ }^{3}$, Karoline Dogum ${ }^{3}$, Aybuke Garipcan ${ }^{1}$, Hannah Meyer ${ }^{1}$, James G. Fox ${ }^{9}$, Eran Elinav ${ }^{10}$, Alper Kucukural ${ }^{11}$, Pawan Kumar ${ }^{12}$, Jeremy McAleer ${ }^{13}$, Christoph A. Thaiss ${ }^{5}$, Aviv Regev ${ }^{3,6,14}$, Stuart H. Orkin $^{2}$ \& Ömer H. Yilmaz ${ }^{3,15}$

${ }^{1}$ Cold Spring Harbor Laboratory, Cold Spring Harbor, New York 11724, USA

2 Division of Hematology/Oncology, Boston Children's Hospital and Department of Pediatric Oncology, Dana-Farber Cancer Institute, Howard Hughes Medical Institute, Harvard Stem Cell Institute, Harvard Medical School, Boston, Massachusetts 02115, USA

${ }^{3}$ The David H. Koch Institute for Integrative Cancer Research at MIT, Department of Biology, MIT, Cambridge, Massachusetts 02139, USA

${ }^{4}$ Department of Medicine, Division of Gastroenterology, Duke University, Durham, NC 27710, USA

${ }^{5}$ Department of Microbiology, Perelman School of Medicine, University of Pennsylvania, Philadelphia, PA 19104, USA

${ }^{6}$ Klarman Cell Observatory, Broad Institute of Harvard and MIT, Cambridge, MA 02142, USA

7 Department of Molecular Biology, Massachusetts General Hospital, Boston, MA 02114, USA

${ }^{8}$ RNA Therapeutics Institute, University of Massachusetts Medical School, Worcester, MA 01605, USA

9 Division of Comparative Medicine, Department of Biological Engineering, Massachusetts Institute of Technology, Cambridge, Massachusetts 02139, USA

${ }^{10}$ Department of Immunology, Weizmann Institute of Science, Rehovot, Israel

${ }^{11}$ Program in Molecular Medicine, University of Massachusetts Medical School, Worcester, MA 01605, USA

12 Department of Microbiology and Immunology, Renaissance School of Medicine, Stony Brook University, Stony Brook, NY, United States

${ }_{13}$ Department of Pharmaceutical Science and Research, Marshall University School of Pharmacy, Huntington, WV, USA

${ }^{14}$ Howard Hughes Medical Institute, Department of Biology, Massachusetts Institute of Technology, Cambridge, MA 02140, USA

${ }^{15}$ Department of Pathology, Massachusetts General Hospital and Harvard Medical School, Boston, MA 02114 USA

${ }^{16}$ These authors contributed equally to this work.

${ }^{17}$ Lead Contact

Correspondence should be addressed to S.B (beyaz@cshl.edu), S.H.O (stuart orkin@dfci.harvard.edu) or Ö.H.Y (ohyilmaz@mit.edu) 


\section{Abstract}

Little is known about how interactions between diet, immune recognition, and intestinal stem cells (ISCs) impact the early steps of intestinal tumorigenesis. Here, we show that a high fat diet (HFD) reduces the expression of the major histocompatibility complex II (MHC-II) genes in ISCs. This decline in ISC MHC-II expression in a HFD correlates with an altered intestinal microbiome composition and is recapitulated in antibiotic treated and germ-free mice on a control diet. Mechanistically, pattern recognition receptor and IFNg signaling regulate MHC-II expression in ISCs. Although MHC-II expression on ISCs is dispensable for stem cell function in organoid cultures in vitro, upon loss of the tumor suppressor gene Apc in a HFD, MHC-II- ISCs harbor greater in vivo tumorinitiating capacity than their MHC-II+ counterparts, thus implicating a role for epithelial MHC-II in suppressing tumorigenesis. Finally, ISC-specific genetic ablation of MHC-II in engineered Apc-mediated intestinal tumor models increases tumor burden in a cell autonomous manner. These findings highlight how a HFD alters the immune recognition properties of ISCs through the regulation of MHC-II expression in a manner that could contribute to intestinal tumorigenesis. 


\section{Introduction}

Diet is a major lifestyle factor that influences health and disease states, including cancer (Beyaz and Yilmaz, 2016; Zitvogel et al., 2017). Significant epidemiologic and preclinical studies link obesity to intestinal cancer incidence. However, how the adaptation of the intestinal epithelium to pro-obesity diets alters the cancer risk remains elusive (Basen-Engquist and Chang, 2011; Calle et al., 2003; Gallagher and LeRoith, 2015). The intestinal epithelium is maintained by $\operatorname{Lgr5}^{+}$intestinal stem cells (ISCs) that reside at the crypt base and give rise to the diverse, specialized cell types of the intestinal lining (Barker et al., 2007). These rapidly renewing ISCs coordinate intestinal adaptation in response to environmental inputs such as diet by balancing stem cell selfrenewal with differentiation divisions (Beyaz et al., 2016; Wang et al., 2018; Yang et al., 2008; Yilmaz et al., 2012). ISCs are also the cells-of-origin for many early intestinal tumors and lie at the interface of dietary nutrients, commensal microbes and immune cells. Thus, understanding how diet induces changes in ISCs and their surrounding components may shed light on the early steps involved in initiation of intestinal cancers (Barker et al., 2009; Belkaid and Hand, 2014; Clevers, 2013; Hooper et al., 2012; Thaiss et al., 2016).

We recently demonstrated that a pro-obesity high fat diet (HFD) enhances intestinal tumorigenicity in part by boosting intestinal stem cell (ISC) and progenitor cell function and increasing the ability of these cells to undergo oncogenic transformation upon loss of the tumor suppressor gene $A p c$ (Beyaz et al., 2016). Because interactions 
between cancer cells and the immune system influence tumor initiation and progression, it is important to understand the crosstalk between tumor-initiating ISCs and immune cells. Although cancers develop several strategies to evade the immune system (Grasso et al., 2018; Liu et al., 2017), little is known about how diet-induced obesity impacts the interaction of ISCs with the immune system at the start of tumor formation.

\section{Results}

\section{HFD dampens MHC-II expression in ISCs}

To explore how a high fat diet (HFD) perturbs immunomodulatory gene expression in ISCs, we examined our previous mRNA-sequencing (RNA-seq) dataset of Lgr5 $^{+}$ISCs isolated from control and HFD-fed mice (Beyaz et al., 2016). We found that ISCs derived from HFD-fed mice significantly downregulate immunomodulatory genes that are involved in the MHC-II pathway (H2-Aa, H2-Ab1, Ciita), anti-microbial response/inflammation (Reg3g, Nfkb2) and costimulation (Icosl, Sectm1a, Sectm1b) (Choi et al., 2011; Dong et al., 2001; Howie et al., 2013; Mukherjee and Hooper, 2015; Tomas et al., 2016) (Fig. 1A, S1A). Given the recently reported heterogeneity within ISCs(Barriga et al., 2017; Biton et al., 2018b), we next performed single-cell RNA-Seq (scRNA-Seq) of ISCs derived from control or HFD mice. Consistent with the bulk RNAseq profiles, H2-Ab1, a key component of MHC-II complex, was among the top 5 differentially expressed genes with > 3-fold higher expression in control compared to HFD ISCs (Fig. 1B, S1B, C) (see methods, MAST test, $p<1 e-10)$ ). To determine the extent of MHC-II downregulation in response to HFD in our SCRNA-seq data, we ranked 
individual ISCs based on their expression pattern of MHC-II pathway genes (MHC-II score, Table 1) and found that HFD ISCs had consistently lower MHC-II score compared to control ISCs (Fig. 1C). We then selected the upper and lower quartiles and performed differential expression analysis between MHC-II low and MHC-II high HFD ISCs. As expected, MHC-II pathway genes (H2-Ab1, Cd74, H2-Aa, H2-DMa) were amongst the top upregulated genes in $\mathrm{MHC}-\mathrm{Il}$ high cells. The genes that were upregulated in MHC-II low cells include the fatty acid-binding protein Fabp2, chaperones Dnajc2 and Hspd1 (Fig. 1D).

Although MHC-II expression and function is generally considered to be restricted to professional antigen presenting cells, several studies have demonstrated that intestinal epithelial cells (IECs) express high levels of MHC-II and are able to capture, process and present antigens to CD4+ T cells, including recent work from our group on ISCs (Biton et al., 2018b; Cerf-Bensussan et al., 1984; Dahan et al., 2007; Hershberg et al., 1997; Telega et al., 2000; Thelemann et al., 2014; Westendorf et al., 2009) (Fig. S1F). We validated the reduction of MHC II expression on IECs and ISCs under a HFD in several ways. In situ hybridization for $\mathrm{H} 2-A b 1$ showed that $\mathrm{MHC}-\mathrm{Il}$ is expressed in the epithelium of control mice epithelium, but was dampened in HFD mice throughout the epithelium, including in the intestinal crypt where ISCs reside (Fig. 1E). Moreover, by flow cytometry, both Epcam+ IECs (Fig. 1F, G) and Lgr5+ ISCs (Fig. 1I, J) expressed high levels of MHC-II protein on their cell surface at steady state, and this expression was significantly downregulated in response to a HFD. Finally, we confirmed that sorted Epcam+ IECs (Fig. 1H) and Lgr5+ ISCs (Fig. 1K) from HFD-fed mice significantly 
downregulated MHC-II expression by qPCR. We then partitioned ISCs into two subpopulations based on their MHC-II expression pattern: MHC-II+ and MHC-II- and assessed their frequencies in control or HFD-fed mice. While in control mice most ISCs were $\mathrm{MHC}-\mathrm{II}_{+}$, a HFD led to a significant reduction in the frequency of MHC-II+ ISCs and to a concomitant increase in the frequency of MHC-II- ISCs (Fig. 1L, M). We sorted these ISC subpopulations to confirm MHC-II expression levels. Consistent with our scRNA-seq analysis, the expression levels of H2-Ab1 mRNA was differentially expressed in these subpopulations both in control and HFD-fed mice, with a significant reduction in response to HFD (Fig. S1D). Altogether, these results indicate HFD leads to suppression of MHC-II expression in the intestinal epithelial cells including ISCs.

\section{PPAR- $\delta$ activation or obesity does not affect MHC-II expression in ISCs}

A HFD perturbs multiple epithelial-intrinsic and -extrinsic pathways that may influence the regulation of MHC-II expression in ISCs(Fu et al., 2019; Schulz et al., 2014). Because our prior findings demonstrated that a PPAR program mediates many of the effects of a HFD in ISCs (Beyaz et al., 2016; Beyaz and Yilmaz, 2016), we next assessed the activation status of a PPAR-ס program in ISCs based on their MHC-II expression pattern in response to a HFD. We found no difference in the expression levels of signature the PPAR- $\delta$ target genes Hmgcs2 and Jag1, or the stem cell marker Lgr5 in MHC-II+ and MHC-II- ISCs in both diet groups (Fig. S1E). Interestingly, agonistinduced PPAR-d activation also did not reduce MHC-II expression in ISCs, indicating that HFD-induced MHC-II downregulation is independent of PPAR- $\delta$ activity in ISCs (Fig. S1I, J). To determine whether MHC-II expression in ISCs is reduced in an 
independent model of obesity, we examined leptin receptor deficient $(\mathrm{db} / \mathrm{db})$ mice, an obesity model that develops on a control diet(Beyaz et al., 2016) (Fig. S1H). Lgr5+ ISCs isolated from both lean control $(\mathrm{db} /+)$ and obese $(\mathrm{db} / \mathrm{db})$ mice expressed high levels of MHC-II, indicating that diet-induced alterations rather than obesity per se inhibits MHC-II expression in ISCs (Fig. S1K, L).

\section{MHC-II expression in ISCs does not influence organoid-forming capacity}

We next assessed whether HFD-mediated downregulation of MHC-II affects stemness. First, we assayed the functional potential of MHC-II+ and MHC-II- ISCs, using an in vitro approach based on the ability of isolated ISCs to form organoid bodies in 3-D culture (Beyaz et al., 2016; Sato et al., 2009a). MHC-Il expression levels in control and HFD ISCs did not affect in vitro organoid formation (Fig. S1F). To further ascertain whether MHC-II expression regulates ISC function, we generated mice with intestinal epithelium-specific MHC-II deletion (vil-iKO; see Methods). Intestinal specific loss of MHC-II did not affect the weight and length of the small intestine or impact crypt depth and villi height (Fig. S2A-G). Furthermore, the proliferation or organoid-forming capacity of ISCs was unaltered upon loss of MHC-II in the intestinal epithelium (Fig. S3A-F). To assess how MHC-II regulates ISC function specifically, we ablated MHC-II in Lgr5 ${ }^{+}$ISCs (Lgr5-iKO; see Methods) and found that loss of MHC-II in ISCs did not affect the capacity of ISCs to initiate organoids (Fig. S3G-H). To determine how MHC-II influences Lgr5+ ISC function in vivo, we performed LacZ lineage tracing analysis and did not detect a significant difference in LacZ+ cells between WT and Lgr5-iKO mice. While these data show that the in vitro organoid-forming capacity of ISCs is 
independent of MHC-II expression and that MHC-II loss does not grossly alter intestinal homeostasis and ISC output (i.e LacZ+ progeny) at steady state, as we previously reported(Biton et al., 2018a) MHC-II may influence ISC differentiation through engaging with T helper cell cytokines in the context of inflammation (Fig. S2I).

\section{MHC-II expression on ISCs depends on the intestinal microbiome}

The intestinal microbiome plays a significant role in regulating intestinal immunity (Belkaid and Hand, 2014; David et al., 2014; Hooper et al., 2012; Round and Mazmanian, 2009; Thaiss et al., 2016). Since dietary perturbations are among the major external factors shaping the intestinal microbiome, we asked whether HFD-induced alterations in the microbiome influence MHC-II expression in ISCs and the intestinal epithelium. Consistent with previous findings, HFD-induced obesity led to microbial dysbiosis with reduced bacterial diversity (Fig. S4A, B) (Ley et al., 2005; Schulz et al., 2014). To determine whether the microbiome is involved in regulation of epithelial MHCII levels, we treated mice with broad-spectrum antibiotics, which ablated bacterial diversity and massiviely altered community composition (Fig. S4A, B). Notably, antibiotic treatment was accompanied by decreased MHC-II expression in ISCs and the intestinal epithelium (Fig. 2A, B), comparable to that observed in HFD. To further corroborate the role of the intestinal microbiome on MHC-II expression on ISCs, we generated germ-free Lgr5-GFP-IRES-CreERT2 mice. ISCs from germ-free mice exhibited reduced MHC-II expression both at RNA and protein levels compared to specific pathogen-free control mice (Fig. 2C-F, S4C, D). 
To gain insight into the spectrum of members of the bacterial microbiome capable of inducing ISC MHC-II expression, we performed comparative 16S rDNA sequencing in HFD-treated mice and controls. Among the bacterial genera most strongly ablated under HFD conditions was Helicobacter (Fig. 2G). To determine whether Helicobacter colonization in mice correlates with the epithelial MHC-II expression in the intestine, we surveyed our mouse facility for presence or absence of Helicobacter species. We determined two separate rooms with mice either naturally colonized with Helicobacter species $(\mathrm{H}+$, dirty room) or without detectable Helicobacter species (H-, clean room) (Fig. 2H). Notably, MHC-II expression was significantly higher in the intestinal epithelium of mice that are housed in dirty room compared to the mice housed in clean room (Fig. 2I). Comparison of microbial composition between dirty mice and clean mice revealed additional bacteria that are more abundant in dirty mice and associate with high epithelial MHC-II expression (Fig. 2J, S4E). These include Odoribacter sp. and Helicobacter sp., which were both ablated in response HFD (Fig. $2 \mathrm{G}, \mathrm{S} 4 \mathrm{E})$. To determine whether colonization of clean mice with the microbial flora of the dirty mice enhances expression of epithelial MHC-II, we co-housed clean mice with dirty mice in the dirty room (Fig. S4G). While clean and dirty mice have distinct microbial composition at baseline as determined by principal component analysis (PCA), after cohousing the microbial profile of clean mice resembles the dirty mice (Fig. S4G). Importantly, co-housing with dirty mice led to a significant increase in Helicobacter sp. abundance in the co-housed clean mice with a concomitant upregulation of epithelial MHC-II compared to mice always remained in the clean room (Fig. 2K-N). These results 
indicate that MHC-II expression in ISCs is regulated by intestinal commensal bacteria including Helicobacter sp., which is altered in response to a HFD.

\section{Pattern recognition receptor (PRR) and IFN $\gamma$ signaling regulate MHC-II expression in ISCs}

Microbiome-induced activation of pattern recognition receptor (PRR) signaling or proinflammatory cytokines induce MHC-II expression in antigen presenting cells and regulate intestinal homeostasis (Abreu, 2010; Rakoff-Nahoum et al., 2004; van den Elsen, 2011). We surveyed PRR expression patterns in ISCs in control and HFD conditions. Consistent with previous reports (Brown et al., 2014; Caruso et al., 2014; Neal et al., 2012; Price et al., 2018), ISCs expressed several Toll-like receptors (TIr1, TIr2, TIr3 and TIr4), as well as Nod-like receptors (Nod1, Nod2) (Fig. 3A). Among these PRRs, TIr2 and Nod2 were downregulated in response to a HFD (Fig. 3A). To test whether activation of TLR2 and NOD2 pathways is sufficient to increase MHC-II expression in ISCs, we treated mice with a dual TLR2/NOD2 agonist (CL429) (Pavot et al., 2014). Indeed, CL429 treatment led to significant upregulation of MHC-II in both control and HFD ISCs, suggesting that signaling through TLR2 and NOD2 can induce MHC-II expression (Fig. 3B, S5A, B). Of note, CL429 treatment did not fully restore MHC-II levels of HFD-treated mice to control mice, which indicates that additional signaling pathways may be perturbed (Fig. 3B, S5A, B). To determine the necessity of PRR signaling through the adaptor protein Myd88 in regulating MHC-II expression, we utilized intestine-specific Myd88-deficient mice (Myd88 iKO) and found significant downregulation of intestinal MHC-II compared to wild-type controls (Fig. S5C, D). 
The intestinal microbiome also influences the activity of proinflammatory cytokine signaling, such as the proinflammatory cytokine interferon-gamma (IFN $\gamma$ ) and downstream JAK1/2 signaling pathways (van den Elsen, 2011). IFN $\gamma$ is a potent inducer of $\mathrm{MHC}-\mathrm{Il}$ expression in antigen presenting cells and other cell types including intestinal epithelial cells (Collins et al., 1984; Thelemann et al., 2014; Wong et al., 1984). We examined the responsiveness to IFN $\gamma$ of MHC-II+ and MHC-II- HFD ISCs, which express similar levels of the IFN $\gamma$ receptors Ifngr1 and Ifngr2 (Fig S1E). IFN $\gamma$ treatment led to upregulation of MHC-II in both MHC-II+ and MHC-II- ISCs (Fig. S5E), suggesting that although HFD ISCs express lower levels of MHC-II, they are still responsive to IFN $\gamma$ stimulation (Choi et al., 2011; Thelemann et al., 2014). To determine whether HFD leads to suppression of epithelial MHC-II expression through dampening IFN $\gamma$ signaling, we assessed the expression levels of IFN $\gamma$-induced genes in control and HFD ISCs. We found ISCs significantly downregulate IFN $\gamma$-induced genes, including MHC-Il pathway genes (H2-Ab1, H2-Aa, Ciita, Cd74, H2-DMa) and upstream pathway genes that regulate MHC-II expression (Stat1, Stat3, Nfkb2, Jak3) in response to HFD (Fig. 3C, D, S5F, G). Of note, the levels of IFN $\gamma$ were significantly reduced in the intestines of HFD mice (Fig. 3E). Notably, administration of a potent JAK1/2 \& TBK1/IKKE inhibitor (CYT378) that inhibits both STAT and NFKB signaling to mice significantly downregulated MHC-II expression in ISCs compared to vehicle-treated controls (Fig. 3E-H, S5H, I) (Tyner et al., 2010). We next asked whether IFN $\gamma$-induced epithelial MHC-II expression is inhibited by CYT378 in in vitro organoid assays. Strikingly, CYT378 blunted the induction of MHC-II expression in response to IFN $\gamma$ (Fig. 
3J). We assessed the necessity of IFN $\gamma$-signaling in regulating MHC-II expression by utilizing IFNGR knockout mice and found dampened epithelial MHC-II expression in IFNGR knockout mice compared to wild-type controls (Figure 3K). HFD-mediated reduction in IFN $\gamma$-inducible and MHC-II pathway gene expression in ISCs posits the possibility that intestinal immune cells may be depleted in the intestinal epithelium. Indeed, we found significant reduction in CD45+ immune cells infiltration to the crypt epithelium, including CD3+, CD8+ and CD4+ T cells (Figure S6A-H). Altogether, these results suggest that PRR and IFN $\gamma$ signaling drive MHC-II expression in the intestinal epithelial cells including ISCs and that HFD attenuates MHC-II expression in ISCs by perturbing these pathways. Our data is consistent with previous studies that demonstrated reduced inflammation and immune infiltration in the intestinal crypt in response to a HFD (Beyaz et al., 2016; Johnson et al., 2015; Schulz et al., 2014).

\section{Dampening MHC-II expression in premalignant ISCs increases intestinal tumorigenesis}

Recognition of antigens by $T$ cells through antigen presentation pathways is a major mechanism for triggering anti-tumor immunity (Vanneman and Dranoff, 2012). Dampening the expression of genes involved in antigen presentation to evade antitumor immune responses is a hallmark of many cancers and correlates with poor prognosis (Lovig et al., 2002; Rimsza et al., 2004; Tarafdar et al., 2017). We found that adenomas arising in HFD-fed mice upon loss of $A p c$ expressed less MHC-II than controls (Fig. S7A, B). To test whether MHC-II expression patterns influence the tumor initiation potential of premalignant ISCs, we studied Lgr5-CreERt2;Apc ${ }^{\mathrm{LL}}$ mice fed a 
control or HFD diet prior to tamoxifen-induced inactivation of the tumor suppressor gene Apc. Whereas premalignant APC ${ }^{\text {null }}$ ISCs in control mice were mostly MHC-II+, APC ${ }^{\text {null }}$ MHC-II- ISCs in HFD mice expressed significantly less MHC-II both at RNA and protein levels (Fig. S7C-F). Interestingly, as we observed in the non-neoplastic intestine (Fig. S3G, H), MHC-II expression levels on APC ${ }^{\text {null }}$ ISCs did not affect their ability to form adenomatous organoids in vitro (Fig. 4A, B). This indicates that decreased MHC-II expression has no effect on the cell-intrinsic oncogenic potential of premalignant APC ${ }^{\text {null }}$ ISCs in organoid cultures in vitro.

To test whether HFD-mediated downregulation of MHC-II in ISCs has protumorigenic effects in vivo under immune surveillance, we utilized a recently developed orthotopic, syngeneic colon transplantation assay in mice (Beyaz et al., 2016). We sorted $A P C^{\text {null }} M H C-I I+$ or APC ${ }^{\text {null }}$ MHC-II- Lgr5-GFP ${ }^{\text {hi }}$ cells by flow cytometry and transplanted them into the colonic submucosa of syngeneic, immune-competent mice. In contrast to the in vitro organoid assay, APC ${ }^{\text {null }}$ MHC-II- Lgr5-GFP ${ }^{\text {hi }}$ cells exhibited greater tumorigenicity when transplanted in vivo than their APC null $\mathrm{MHC}-\mathrm{II}+$ counterparts (Fig. 4C, S7G-I). Notably, when transplanted to immunodeficient hosts that lack


to equal numbers of tumors, highlighting the significance of $\mathrm{MHC}$-II recognition by immune cells in controlling intestinal tumor initiation (Fig. 4D, S7J-L). Finally, to ascertain the role of epithelial MHC-II expression during intestinal tumorigenesis, we generated two genetic mouse models. First, using epithelial-specific inducible deletion model of MHC-II together with tumor suppressor Apc (vilCreERt2; Apc ${ }^{\mathrm{L} / \mathrm{L}} ; \mathrm{MHC}-\mathrm{II}^{\mathrm{L} / \mathrm{L}}$ ), we 
initated single tumors in the distal colon by administering 4-OHT with our endoscopyguided injection system. We found that mice with MHC-II KO allele (vil-Cre-ERt2 APC ${ }^{\mathrm{L} / \mathrm{L}}$, MHC-II/+) gave rise to larger tumors compared to mice with WT allele (vil-Cre-ERt2 $\mathrm{APC}^{\mathrm{LL}}, \mathrm{MHC}^{-\mathrm{II}^{\mathrm{L}+}}$ ) (Fig 4E, F). Second, we initiated tumors using Lgr5+ ISC-specific inducible deletion model of MHC-II together with one copy of the tumor suppressor ApC that leads to intestinal tumor formation due to loss of heterozygosity. While we did not detect significant difference in the numbers of T cells inflitrating the tumors at the time of analysis, we found that specific loss of MHC-II in ISCs was associated with greater numbers of intestinal tumors compared to their MHC-II-proficient counterparts (Fig. 4G, S7M,O). These results illustrate that HFD-mediated reduction of MHC-II in premalignant ISCs enhances tumor initiation.

\section{Discussion}

ISCs self-renew and differentiate into cells that constitute the intestinal epithelium, which is continuously replenished (Clevers, 2013). Acquisition of oncogenic mutations in these rapidly cycling stem cells leads to tumors that are subject to clearance by T cells (Agudo et al., 2018; Barker et al., 2009; Schepers et al., 2012). It is also becoming increasingly evident that the crosstalk between tissue stem cells and immune cells influences differentiation, homeostasis and cancer risk (Ali et al., 2017; Biton et al., 2018b; Chakrabarti et al., 2018; Hoytema van Konijnenburg et al., 2017; Lindemans et al., 2015; Naik et al., 2018; Naik et al., 2017). Here, we find that a HFD dampens MHC-II expression in ISCs which can promote intestinal cancer by impacting immune surveillance through MHC II. While MHC-I antigen presentation pathway- 
mediated activation of cytotoxic $\mathrm{CD}^{+} \mathrm{T}$ cells is frequently studied in the context of antitumor immune responses, $\mathrm{MHC}$-II-mediated activation of $\mathrm{CD}^{+} \mathrm{T}$ cells is also pivotal for tumor immunity (Hung et al., 1998; Takeuchi and Saito, 2017; Tran et al., 2014; Wang, 2001; Xie et al., 2010; Zhang et al., 2009). Indeed, recent studies have demonstrated that $\mathrm{MHC}$-II-restricted $\mathrm{CD}^{+}{ }^{+} \mathrm{T}$ cells are able to eradicate tumors both directly and indirectly through the licensing of dendritic cells and helping $\mathrm{CD}^{+} \mathrm{T}$ cell responses (Haabeth et al., 2016; Hirschhorn-Cymerman et al., 2012; Kreiter et al., 2015; Lu et al., 2017; Spitzer et al., 2017; Tran et al., 2014). Consistent with previous studies demonstrating that tumors downregulate $\mathrm{MHC}$-II expression to escape from immune surveillance (Park et al., 2017; Tarafdar et al., 2017), our data implicate dietary regulation of $\mathrm{MHC}$-II expression in ISCs as playing a critical role in intestinal tumorigenesis. Whether diet-induced reduction in MHC-II expression in ISCs directly or indirectly affects anti-tumor $\mathrm{CD}^{+} \mathrm{T}$ cell responses warrants further investigation.

We previously demonstrated that the activation of PPAR-d by the lipid constituents of a HFD is an ISC cell-intrinsic mechanism that enhances tumorigenicity in the intestine (Beyaz et al., 2016). The findings presented here indicate that downregulation of MHC-II in ISCs in response to a HFD provides an orthogonal mechanism contributing to intestinal cancer. Indeed, a recent large scale genome-wide variant scan for colorectal cancer identified variants associated with cancer risk in $\mathrm{MHC}$ Il gene loci(Huyghe et al., 2018). Together, our results highlight how diet impacts multiple mechanisms that modify cancer risk in the intestine. Because a HFD influences cancer incidence in both mucosal and non-mucosal tissues (Beyaz et al., 2016; Chen et 
al., 2018; Kroenke et al., 2013; Pascual et al., 2017; Schulz et al., 2014; Yang et al., 2017; Yang et al., 2008), it will be important to explore whether MHC-II mediated immune surveillance of stem cells also takes place in other tissues and whether it is affected by dietary perturbations. 


\section{REFERENCES}

Abreu, M.T. (2010). Toll-like receptor signalling in the intestinal epithelium: how bacterial recognition shapes intestinal function. Nat Rev Immunol 10, 131-144.

Agudo, J., Park, E.S., Rose, S.A., Alibo, E., Sweeney, R., Dhainaut, M., Kobayashi, K.S., Sachidanandam, R., Baccarini, A., Merad, M., et al. (2018). Quiescent Tissue Stem Cells Evade Immune Surveillance. Immunity 48, 271-285 e275.

Ali, N., Zirak, B., Rodriguez, R.S., Pauli, M.L., Truong, H.A., Lai, K., Ahn, R., Corbin, K., Lowe, M.M., Scharschmidt, T.C., et al. (2017). Regulatory T Cells in Skin Facilitate Epithelial Stem Cell Differentiation. Cell 169, 1119-1129 e1111.

Barker, N., Ridgway, R.A., van Es, J.H., van de Wetering, M., Begthel, H., van den Born, M., Danenberg, E., Clarke, A.R., Sansom, O.J., and Clevers, H. (2009). Crypt stem cells as the cells-of-origin of intestinal cancer. Nature 457, 608-611.

Barker, N., van Es, J.H., Kuipers, J., Kujala, P., van den Born, M., Cozijnsen, M., Haegebarth, A., Korving, J., Begthel, H., Peters, P.J., et al. (2007). Identification of stem cells in small intestine and colon by marker gene Lgr5. Nature 449, 1003-1007.

Barriga, F.M., Montagni, E., Mana, M., Mendez-Lago, M., Hernando-Momblona, X., Sevillano, M., Guillaumet-Adkins, A., Rodriguez-Esteban, G., Buczacki, S.J.A., Gut, M., et al. (2017). Mex3a Marks a Slowly Dividing Subpopulation of Lgr5+ Intestinal Stem Cells. Cell Stem Cell 20, 801-816 e807.

Basen-Engquist, K., and Chang, M. (2011). Obesity and cancer risk: recent review and evidence. Curr Oncol Rep 13, 71-76.

Belkaid, Y., and Hand, T.W. (2014). Role of the microbiota in immunity and inflammation. Cell 157, 121-141.

Beyaz, S., Mana, M.D., Roper, J., Kedrin, D., Saadatpour, A., Hong, S.J., Bauer-Rowe, K.E., Xifaras, M.E., Akkad, A., Arias, E., et al. (2016). High-fat diet enhances stemness and tumorigenicity of intestinal progenitors. Nature 531, 53-58.

Beyaz, S., and Yilmaz, O.H. (2016). Molecular Pathways: Dietary Regulation of Stemness and Tumor Initiation by the PPAR-delta Pathway. Clin Cancer Res 22, 5636-5641.

Biton, M., Haber, A.L., Rogel, N., Burgin, G., Beyaz, S., Schnell, A., Ashenberg, O., Su, C.W., Smillie, C., Shekhar, K., et al. (2018). T Helper Cell Cytokines Modulate Intestinal Stem Cell Renewal and Differentiation. Cell.

Brown, M., Hughes, K.R., Moossavi, S., Robins, A., and Mahida, Y.R. (2014). Toll-like receptor expression in crypt epithelial cells, putative stem cells and intestinal myofibroblasts isolated from controls and patients with inflammatory bowel disease. Clin Exp Immunol 178, 28-39.

Calle, E.E., Rodriguez, C., Walker-Thurmond, K., and Thun, M.J. (2003). Overweight, obesity, and mortality from cancer in a prospectively studied cohort of U.S. adults. N Engl J Med 348, 1625-1638.

Caruso, R., Warner, N., Inohara, N., and Nunez, G. (2014). NOD1 and NOD2: signaling, host defense, and inflammatory disease. Immunity 41, 898-908.

Cerf-Bensussan, N., Quaroni, A., Kurnick, J.T., and Bhan, A.K. (1984). Intraepithelial lymphocytes modulate Ia expression by intestinal epithelial cells. J Immunol 132, 22442252. 
Chakrabarti, R., Celia-Terrassa, T., Kumar, S., Hang, X., Wei, Y., Choudhury, A., Hwang, J., Peng, J., Nixon, B., Grady, J.J., et al. (2018). Notch ligand Dll1 mediates cross-talk between mammary stem cells and the macrophageal niche. Science 360.

Chen, M., Zhang, J., Sampieri, K., Clohessy, J.G., Mendez, L., Gonzalez-Billalabeitia, E., Liu, X.S., Lee, Y.R., Fung, J., Katon, J.M., et al. (2018). An aberrant SREBP-dependent lipogenic program promotes metastatic prostate cancer. Nat Genet 50, 206-218.

Choi, N.M., Majumder, P., and Boss, J.M. (2011). Regulation of major histocompatibility complex class II genes. Curr Opin Immunol 23, 81-87.

Clevers, H. (2013). The intestinal crypt, a prototype stem cell compartment. Cell 154, 274284.

Collins, T., Korman, A.J., Wake, C.T., Boss, J.M., Kappes, D.J., Fiers, W., Ault, K.A., Gimbrone, M.A., Jr., Strominger, J.L., and Pober, J.S. (1984). Immune interferon activates multiple class II major histocompatibility complex genes and the associated invariant chain gene in human endothelial cells and dermal fibroblasts. Proc Natl Acad Sci U S A 81, 4917-4921.

Dahan, S., Roth-Walter, F., Arnaboldi, P., Agarwal, S., and Mayer, L. (2007). Epithelia: lymphocyte interactions in the gut. Immunol Rev 215, 243-253.

David, L.A., Maurice, C.F., Carmody, R.N., Gootenberg, D.B., Button, J.E., Wolfe, B.E., Ling, A.V., Devlin, A.S., Varma, Y., Fischbach, M.A., et al. (2014). Diet rapidly and reproducibly alters the human gut microbiome. Nature 505, 559-563.

Dong, C., Juedes, A.E., Temann, U.A., Shresta, S., Allison, J.P., Ruddle, N.H., and Flavell, R.A. (2001). ICOS co-stimulatory receptor is essential for T-cell activation and function. Nature 409, 97-101.

Fu, T., Coulter, S., Yoshihara, E., Oh, T.G., Fang, S., Cayabyab, F., Zhu, Q., Zhang, T., Leblanc, M., Liu, S., et al. (2019). FXR Regulates Intestinal Cancer Stem Cell Proliferation. Cell 176, 1098-1112 e1018.

Gallagher, E.J., and LeRoith, D. (2015). Obesity and Diabetes: The Increased Risk of Cancer and Cancer-Related Mortality. Physiol Rev 95, 727-748.

Grasso, C.S., Giannakis, M., Wells, D.K., Hamada, T., Mu, X.J., Quist, M., Nowak, J.A., Nishihara, R., Qian, Z.R., Inamura, K., et al. (2018). Genetic mechanisms of immune evasion in colorectal cancer. Cancer Discov.

Haabeth, O.A., Tveita, A., Fauskanger, M., Hennig, K., Hofgaard, P.0., and Bogen, B. (2016). Idiotype-specific CD4(+) T cells eradicate disseminated myeloma. Leukemia 30, 1216-1220. Hershberg, R.M., Framson, P.E., Cho, D.H., Lee, L.Y., Kovats, S., Beitz, J., Blum, J.S., and Nepom, G.T. (1997). Intestinal epithelial cells use two distinct pathways for HLA class II antigen processing. J Clin Invest 100, 204-215.

Hirschhorn-Cymerman, D., Budhu, S., Kitano, S., Liu, C., Zhao, F., Zhong, H., Lesokhin, A.M., Avogadri-Connors, F., Yuan, J., Li, Y., et al. (2012). Induction of tumoricidal function in CD4+ T cells is associated with concomitant memory and terminally differentiated phenotype. J Exp Med 209, 2113-2126.

Hooper, L.V., Littman, D.R., and Macpherson, A.J. (2012). Interactions between the microbiota and the immune system. Science 336, 1268-1273.

Howie, D., Garcia Rueda, H., Brown, M.H., and Waldmann, H. (2013). Secreted and transmembrane $1 \mathrm{~A}$ is a novel co-stimulatory ligand. PLoS One 8, e73610.

Hoytema van Konijnenburg, D.P., Reis, B.S., Pedicord, V.A., Farache, J., Victora, G.D., and Mucida, D. (2017). Intestinal Epithelial and Intraepithelial T Cell Crosstalk Mediates a Dynamic Response to Infection. Cell 171, 783-794 e713. 
Hung, K., Hayashi, R., Lafond-Walker, A., Lowenstein, C., Pardoll, D., and Levitsky, H. (1998). The central role of CD4(+) T cells in the antitumor immune response. J Exp Med 188, 23572368.

Huyghe, J.R., Bien, S.A., Harrison, T.A., Kang, H.M., Chen, S., Schmit, S.L., Conti, D.V., Qu, C., Jeon, J., Edlund, C.K., et al. (2018). Discovery of common and rare genetic risk variants for colorectal cancer. Nat Genet.

Johnson, A.M., Costanzo, A., Gareau, M.G., Armando, A.M., Quehenberger, O., Jameson, J.M., and Olefsky, J.M. (2015). High fat diet causes depletion of intestinal eosinophils associated with intestinal permeability. PloS one 10, e0122195.

Kambayashi, T., and Laufer, T.M. (2014). Atypical MHC class II-expressing antigenpresenting cells: can anything replace a dendritic cell? Nat Rev Immunol 14, 719-730.

Kreiter, S., Vormehr, M., van de Roemer, N., Diken, M., Lower, M., Diekmann, J., Boegel, S., Schrors, B., Vascotto, F., Castle, J.C., et al. (2015). Mutant MHC class II epitopes drive therapeutic immune responses to cancer. Nature 520, 692-696.

Kroenke, C.H., Kwan, M.L., Sweeney, C., Castillo, A., and Caan, B.J. (2013). High- and low-fat dairy intake, recurrence, and mortality after breast cancer diagnosis. J Natl Cancer Inst 105, 616-623.

Ley, R.E., Backhed, F., Turnbaugh, P., Lozupone, C.A., Knight, R.D., and Gordon, J.I. (2005). Obesity alters gut microbial ecology. Proc Natl Acad Sci U S A 102, 11070-11075.

Lindemans, C.A., Calafiore, M., Mertelsmann, A.M., O'Connor, M.H., Dudakov, J.A., Jenq, R.R., Velardi, E., Young, L.F., Smith, 0.M., Lawrence, G., et al. (2015). Interleukin-22 promotes intestinal-stem-cell-mediated epithelial regeneration. Nature 528, 560-564.

Liu, L., Nishihara, R., Qian, Z.R., Tabung, F.K., Nevo, D., Zhang, X., Song, M., Cao, Y., Mima, K., Masugi, Y., et al. (2017). Association Between Inflammatory Diet Pattern and Risk of Colorectal Carcinoma Subtypes Classified by Immune Responses to Tumor. Gastroenterology 153, 1517-1530 e1514.

Lovig, T., Andersen, S.N., Thorstensen, L., Diep, C.B., Meling, G.I., Lothe, R.A., and Rognum, T.0. (2002). Strong HLA-DR expression in microsatellite stable carcinomas of the large bowel is associated with good prognosis. Br J Cancer 87, 756-762.

Lu, Y.C., Parker, L.L., Lu, T., Zheng, Z., Toomey, M.A., White, D.E., Yao, X., Li, Y.F., Robbins, P.F., Feldman, S.A., et al. (2017). Treatment of Patients With Metastatic Cancer Using a Major Histocompatibility Complex Class II-Restricted T-Cell Receptor Targeting the Cancer Germline Antigen MAGE-A3. J Clin Oncol 35, 3322-3329.

Mukherjee, S., and Hooper, L.V. (2015). Antimicrobial defense of the intestine. Immunity 42, 28-39.

Naik, S., Larsen, S.B., Cowley, C.J., and Fuchs, E. (2018). Two to Tango: Dialog between Immunity and Stem Cells in Health and Disease. Cell 175, 908-920.

Naik, S., Larsen, S.B., Gomez, N.C., Alaverdyan, K., Sendoel, A., Yuan, S., Polak, L., Kulukian, A., Chai, S., and Fuchs, E. (2017). Inflammatory memory sensitizes skin epithelial stem cells to tissue damage. Nature 550, 475-480.

Neal, M.D., Sodhi, C.P., Jia, H., Dyer, M., Egan, C.E., Yazji, I., Good, M., Afrazi, A., Marino, R., Slagle, D., et al. (2012). Toll-like receptor 4 is expressed on intestinal stem cells and regulates their proliferation and apoptosis via the p53 up-regulated modulator of apoptosis. J Biol Chem 287, 37296-37308.

Park, I.A., Hwang, S.H., Song, I.H., Heo, S.H., Kim, Y.A., Bang, W.S., Park, H.S., Lee, M., Gong, G., and Lee, H.J. (2017). Expression of the MHC class II in triple-negative breast cancer is 
associated with tumor-infiltrating lymphocytes and interferon signaling. PLoS One 12, e0182786.

Pascual, G., Avgustinova, A., Mejetta, S., Martin, M., Castellanos, A., Attolini, C.S., Berenguer, A., Prats, N., Toll, A., Hueto, J.A., et al. (2017). Targeting metastasis-initiating cells through the fatty acid receptor CD36. Nature 541, 41-45.

Pavot, V., Rochereau, N., Resseguier, J., Gutjahr, A., Genin, C., Tiraby, G., Perouzel, E., Lioux, T., Vernejoul, F., Verrier, B., et al. (2014). Cutting edge: New chimeric NOD2/TLR2 adjuvant drastically increases vaccine immunogenicity. J Immunol 193, 5781-5785.

Price, A.E., Shamardani, K., Lugo, K.A., Deguine, J., Roberts, A.W., Lee, B.L., and Barton, G.M. (2018). A Map of Toll-like Receptor Expression in the Intestinal Epithelium Reveals Distinct Spatial, Cell Type-Specific, and Temporal Patterns. Immunity 49, 560-575 e566.

Rakoff-Nahoum, S., Paglino, J., Eslami-Varzaneh, F., Edberg, S., and Medzhitov, R. (2004). Recognition of commensal microflora by toll-like receptors is required for intestinal homeostasis. Cell 118, 229-241.

Rimsza, L.M., Roberts, R.A., Miller, T.P., Unger, J.M., LeBlanc, M., Braziel, R.M., Weisenberger, D.D., Chan, W.C., Muller-Hermelink, H.K., Jaffe, E.S., et al. (2004). Loss of MHC class II gene and protein expression in diffuse large B-cell lymphoma is related to decreased tumor immunosurveillance and poor patient survival regardless of other prognostic factors: a follow-up study from the Leukemia and Lymphoma Molecular Profiling Project. Blood 103, 4251-4258.

Round, J.L., and Mazmanian, S.K. (2009). The gut microbiota shapes intestinal immune responses during health and disease. Nat Rev Immunol 9, 313-323.

Sato, T., Vries, R.G., Snippert, H.J., van de Wetering, M., Barker, N., Stange, D.E., van Es, J.H., Abo, A., Kujala, P., Peters, P.J., et al. (2009). Single Lgr5 stem cells build crypt-villus structures in vitro without a mesenchymal niche. Nature 459, 262-265.

Schepers, A.G., Snippert, H.J., Stange, D.E., van den Born, M., van Es, J.H., van de Wetering, M., and Clevers, H. (2012). Lineage tracing reveals Lgr5+ stem cell activity in mouse intestinal adenomas. Science 337, 730-735.

Schulz, M.D., Atay, C., Heringer, J., Romrig, F.K., Schwitalla, S., Aydin, B., Ziegler, P.K., Varga, J., Reindl, W., Pommerenke, C., et al. (2014). High-fat-diet-mediated dysbiosis promotes intestinal carcinogenesis independently of obesity. Nature 514, 508-512.

Spitzer, M.H., Carmi, Y., Reticker-Flynn, N.E., Kwek, S.S., Madhireddy, D., Martins, M.M., Gherardini, P.F., Prestwood, T.R., Chabon, J., Bendall, S.C., et al. (2017). Systemic Immunity Is Required for Effective Cancer Immunotherapy. Cell 168, 487-502 e415.

Takeuchi, A., and Saito, T. (2017). CD4 CTL, a Cytotoxic Subset of CD4(+) T Cells, Their Differentiation and Function. Front Immunol 8, 194.

Tarafdar, A., Hopcroft, L.E., Gallipoli, P., Pellicano, F., Cassels, J., Hair, A., Korfi, K., Jorgensen, H.G., Vetrie, D., Holyoake, T.L., et al. (2017). CML cells actively evade host immune surveillance through cytokine-mediated downregulation of MHC-II expression. Blood 129, 199-208.

Telega, G.W., Baumgart, D.C., and Carding, S.R. (2000). Uptake and presentation of antigen to $\mathrm{T}$ cells by primary colonic epithelial cells in normal and diseased states. Gastroenterology 119, 1548-1559.

Thaiss, C.A., Itav, S., Rothschild, D., Meijer, M., Levy, M., Moresi, C., Dohnalova, L., Braverman, S., Rozin, S., Malitsky, S., et al. (2016). Persistent microbiome alterations modulate the rate of post-dieting weight regain. Nature. 
Thelemann, C., Eren, R.O., Coutaz, M., Brasseit, J., Bouzourene, H., Rosa, M., Duval, A., Lavanchy, C., Mack, V., Mueller, C., et al. (2014). Interferon-gamma induces expression of MHC class II on intestinal epithelial cells and protects mice from colitis. PLoS One 9, e86844.

Tomas, J., Mulet, C., Saffarian, A., Cavin, J.B., Ducroc, R., Regnault, B., Kun Tan, C., Duszka, K., Burcelin, R., Wahli, W., et al. (2016). High-fat diet modifies the PPAR-gamma pathway leading to disruption of microbial and physiological ecosystem in murine small intestine. Proc Natl Acad Sci U S A 113, E5934-E5943.

Tran, E., Turcotte, S., Gros, A., Robbins, P.F., Lu, Y.C., Dudley, M.E., Wunderlich, J.R., Somerville, R.P., Hogan, K., Hinrichs, C.S., et al. (2014). Cancer immunotherapy based on mutation-specific CD4+ T cells in a patient with epithelial cancer. Science 344, 641-645.

Tyner, J.W., Bumm, T.G., Deininger, J., Wood, L., Aichberger, K.J., Loriaux, M.M., Druker, B.J., Burns, C.J., Fantino, E., and Deininger, M.W. (2010). CYT387, a novel JAK2 inhibitor, induces hematologic responses and normalizes inflammatory cytokines in murine myeloproliferative neoplasms. Blood 115, 5232-5240.

van den Elsen, P.J. (2011). Expression regulation of major histocompatibility complex class I and class II encoding genes. Front Immunol 2, 48.

Vanneman, M., and Dranoff, G. (2012). Combining immunotherapy and targeted therapies in cancer treatment. Nat Rev Cancer 12, 237-251.

Wang, B., Rong, X., Palladino, E.N.D., Wang, J., Fogelman, A.M., Martin, M.G., Alrefai, W.A., Ford, D.A., and Tontonoz, P. (2018). Phospholipid Remodeling and Cholesterol Availability Regulate Intestinal Stemness and Tumorigenesis. Cell stem cell 22, 206-220 e204.

Wang, R.F. (2001). The role of MHC class II-restricted tumor antigens and CD4+ T cells in antitumor immunity. Trends Immunol 22, 269-276.

Westendorf, A.M., Fleissner, D., Groebe, L., Jung, S., Gruber, A.D., Hansen, W., and Buer, J. (2009). CD4+Foxp3+ regulatory $\mathrm{T}$ cell expansion induced by antigen-driven interaction with intestinal epithelial cells independent of local dendritic cells. Gut 58, 211-219.

Wong, G.H., Bartlett, P.F., Clark-Lewis, I., Battye, F., and Schrader, J.W. (1984). Inducible expression of $\mathrm{H}-2$ and la antigens on brain cells. Nature 310, 688-691.

Xie, Y., Akpinarli, A., Maris, C., Hipkiss, E.L., Lane, M., Kwon, E.K., Muranski, P., Restifo, N.P., and Antony, P.A. (2010). Naive tumor-specific CD4(+) T cells differentiated in vivo eradicate established melanoma. J Exp Med 207, 651-667.

Yang, J.J., Yu, D., Takata, Y., Smith-Warner, S.A., Blot, W., White, E., Robien, K., Park, Y., Xiang, Y.B., Sinha, R., et al. (2017). Dietary Fat Intake and Lung Cancer Risk: A Pooled Analysis. J Clin Oncol 35, 3055-3064.

Yang, K., Kurihara, N., Fan, K., Newmark, H., Rigas, B., Bancroft, L., Corner, G., Livote, E., Lesser, M., Edelmann, W., et al. (2008). Dietary induction of colonic tumors in a mouse model of sporadic colon cancer. Cancer Res 68, 7803-7810.

Yilmaz, O.H., Katajisto, P., Lamming, D.W., Gultekin, Y., Bauer-Rowe, K.E., Sengupta, S., Birsoy, K., Dursun, A., Yilmaz, V.0., Selig, M., et al. (2012). mTORC1 in the Paneth cell niche couples intestinal stem-cell function to calorie intake. Nature 486, 490-495.

Zhang, S., Zhang, H., and Zhao, J. (2009). The role of CD4 T cell help for CD8 CTL activation. Biochem Biophys Res Commun 384, 405-408.

Zitvogel, L., Pietrocola, F., and Kroemer, G. (2017). Nutrition, inflammation and cancer. Nat Immunol 18, 843-850. 


\section{Materials and Methods}

\section{Mice, High Fat Diet, and drug treatment}

Mice were housed in the Cold Spring Harbor Laboratory and Koch Institute for Integrative Cancer Research. The following strains were obtained from the Jackson Laboratory: Lgr5-EGFP-IRES-CreERT2 (strain name: B6.129P2-Lgr5 ${ }^{\mathrm{tm} 1 \text { (cre/ERT2)Cle} / J, ~}$

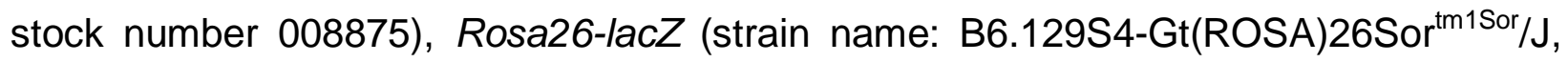
stock number 003474), db/db (strain name: B6.BKS(D)-Lepr ${ }^{\mathrm{b}} / \mathrm{J}$, stock number 000697),

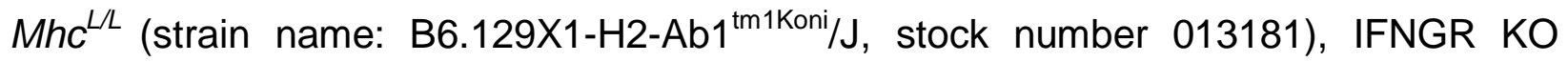
(C57BL/6N-Ifngr1tm1.2Rds/J, stock number 025545. Apc ${ }^{\text {loxp exon } 14}\left(\mathrm{Apc}^{\mathrm{L} / \mathrm{L}}\right)$ has been previously described(Colnot et al., 2004). Villin-CreERT2 was a gift from Sylvie Robine. Diet-induced obesity studies were performed by using a high fat diet consisting of 60 $\mathrm{kcal} \%$ fat (Research Diets D12492) beginning at the age of 8-12 weeks and extending for 9 to 14 months. Control mice were age- and sex-matched and were fed matched purified control diet (Research Diets, D12450J). GW501516 (Enzo) was reconstituted in DMSO at $4.5 \square \mathrm{mg}_{\square} \mathrm{ml}^{-1}$ and diluted 1:10 in a solution of 5\% PEG400 (Hampton Research), 5\% Tween80 (Sigma), 90\% $\mathrm{H}_{2} \mathrm{O}$ (injection buffer) for a daily intraperitoneal injection of $4 \mathrm{mg} \mathrm{kg}^{-1}$. Apc exon 14 was excised by tamoxifen suspended in sunflower seed oil (Spectrum S1929) at a concentration of $10 \mathrm{mg} \mathrm{ml}^{-1}$ and $250 \mu \mathrm{l}$ per $25 \mathrm{~g}$ of body weight, and administered by intraperitoneal injection twice over 4 days before harvesting tissue. BrdU (Sigma) was prepared at $10 \mathrm{mg} \mathrm{ml}^{-1}$ in PBS, passed through $0.22 \mu \mathrm{m}$ filter and injected at $100 \mathrm{mg} \mathrm{kg}^{-1}$. CYT387 (SelleckChem) was reconstituted in DMSO at $10 \mathrm{mg} \mathrm{ml}^{-1}$ and diluted 1:100 in injection buffer for a daily gavage of $25 \mathrm{mg} \mathrm{kg}^{-1}$ 
for 7 days. CL429 (InvivoGen) was reconstituted in DMSO at $5 \mathrm{mg} \mathrm{ml}^{-1}$ and diluted 1:20 in a solution of injection buffer for a daily gavage of $2 \mathrm{mg} \mathrm{kg}^{-1}$ for 7 days. For broadspectrum antibiotic treatment, mice received a mixture of vacomycin $(0.5 \mathrm{~g} / \mathrm{l})$, ampicillin $(1 \mathrm{~g} / \mathrm{l})$, metronidazole $(1 \mathrm{~g} / \mathrm{l})$ and neomycin $(1 \mathrm{~g} / \mathrm{l})$ in the drinking water.

\section{Immunohistochemistry (IHC) and immunofluorescence (IF)}

As previously described(Yilmaz et al., 2012), tissues were fixed in $10 \%$ formalin, paraffin embedded and sectioned. Antigen retrieval was performed with Borg Decloaker RTU solution (Biocare Medical) in a pressurized Decloaking Chamber (Biocare Medical) for $3 \sqcap$ minutes. Antibodies used: rat anti-BrdU (1:2000, Abcam 6326), mouse monoclonal $\beta$-catenin (1:100, BD Biosciences 610154), rabbit monoclonal OLFM4 (1:10,000, gift from CST, clone PP7), Biotin-conjugated secondary donkey anti-rabbit or anti-rat antibodies were used from Jackson ImmunoResearch. The Vectastain Elite ABC immunoperoxidase detection kit (Vector Labs) followed by Dako Liquid DAB+ Substrate (Dako) was used for visualization. All antibody incubations involving tissue or sorted cells were performed with Common Antibody Diluent (Cell Signaling).

\section{In situ hybridization}

Single-molecule in situhybridization was performed to detect MHC-II ( $\mathrm{H} 2-\mathrm{Ab} 1$, \#414731) using Advanced Cell Diagnostics RNAscope 2.5 HD Detection Kit following manufacturer's instructions. 


\section{Flow cytometry and isolation of ISCs and Paneth cells}

As previously reported and briefly summarized here, small intestines and colons were removed, washed with cold PBS-/-, opened laterally and cut into 3-5mm fragments. Pieces were washed multiple times with cold PBS-/- until clean, washed 2-3 with PBS-//EDTA (10mM), and incubated on ice for 90-120 minutes while mixing at 30-minute intervals. Crypts were then mechanically separated from the connective tissue by shaking, and filtered through a $70-\mu \mathrm{m}$ mesh into a $50-\mathrm{ml}$ conical tube to remove villus material (for small intestine) and tissue fragments. Crypts were removed from this step for crypt culture experiments and embedded in Matrigel ${ }^{\mathrm{TM}}$ with crypt culture media. For ISC isolation, the crypt suspensions were dissociated to individual cells with TrypLE Express (Invitrogen). Cell labeling consisted of an antibody cocktail comprising IA-PE (eBioscience, 30-F11, 1:500), EPCAM APC (eBioscience, G8.8, 1:100), CD11b-APCCy7 (eBioscience, 1:500), and CD45 Pacific Blue (eBioscience, 1:500). Dead cells were excluded from the analysis with the viability dye 7-AAD (Life Technologies). ISCs were isolated as Lgr5-EGFP ${ }^{\text {hi }}$ Epcam ${ }^{+} \mathrm{CD}^{-} 5^{-} 7-\mathrm{AAD}^{-}$with a BD FACS Aria II SORP cell sorter into supplemented crypt culture medium for culture.

\section{Culture media for crypts and isolated cells}

Isolated crypts were counted and embedded in Matrigel ${ }^{\mathrm{TM}}$ (Corning 356231 growth factor reduced) at 5-10 crypts per $\mu \mathrm{l}$ and cultured in a modified form of medium as described previously(Sato et al., 2009b). Unless otherwise noted, Advanced DMEM (Gibco) was supplemented by $\quad$ EGF $\quad 40 \square \mathrm{ng} \square \mathrm{ml}^{-1}(\mathrm{R} \& \mathrm{D}), \quad$ Noggin 
$200 \square \mathrm{ng} \square \mathrm{ml}^{-1}$ (Peprotech), R-spondin $500 \square \mathrm{ng} \square \mathrm{ml}^{-1}$ (R\&D or Sino Biological), N-acetyl-

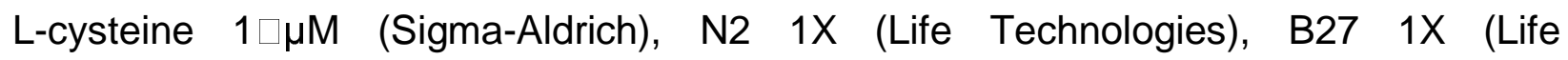
Technologies), Chiron $10 \mu \mathrm{M}$ (Stemgent), Y-27632 dihydrochloride monohydrate $20 \square$ ng $\mathrm{ml}^{-1}$ (Sigma-Aldrich). $25 \square \mu \mathrm{L}$ drops of Matrigel ${ }^{\mathrm{TM}}$ with crypts were plated onto a flat bottom 48-well plate (Corning 3548) and allowed to solidify for $20 \square$ minutes in a $37^{\circ} \mathrm{C}$ incubator. Three hundred microliters of crypt culture medium was then overlaid onto the Matrige ${ }^{\mathrm{TM}}$, changed every three days, and maintained at $37^{\circ} \mathrm{C}$ in fully humidified chambers containing $5 \% \mathrm{CO}_{2}$. Clonogenicity (colony-forming efficiency) was calculated by plating $50-300$ crypts per well and assessing organoid formation $3-7 \square$ days or as specified after initiation of cultures.

Isolated ISCs or progenitor cells were centrifuged for $5 \square$ minutes at $250 \mathrm{~g}$, re-suspended in the appropriate volume of crypt culture medium (500-1,000 $\square$ cells $\left.\left.\square \mu\right|^{-1}\right)$, then seeded onto 25-30 $\mu \mathrm{M} \mathrm{Matrige}^{\mathrm{TM}}$ (Corning 356231 growth factor reduced) containing $1 \square \mu \mathrm{M}$ Jagged (Ana-Spec) in a flat bottom 48-well plate (Corning 3548). Alternatively, ISCs and Paneth cells were mixed after sorting in a 1:1 ratio, centrifuged, and then seeded onto Matrigel $^{\mathrm{TM}}$. The Matrigel ${ }^{\mathrm{TM}}$ and cells were allowed to solidify before adding $300 \mu \mathrm{l}$ of crypt culture medium. The crypt media was changed every second or third day. Organoid bodies were quantified on days 3,7 and 10 of culture, unless otherwise specified. In secondary experiments, individual primary organoids were mechanically dissociated and replated, or organoids were dissociated for 10 minutes in TrypLE Express at $32^{\circ} \mathrm{C}$, resuspended with SMEM (Life Technologies), centrifuged and resuspended in cold SMEM with viability dye 7-AAD. Live cells were sorted and seeded onto Matrigel ${ }^{\mathrm{TM}}$ as previously described. 


\section{Bulk RNA-Seq}

RNA Isolation. For RNA-seq, total RNA was extracted from 200K sorted Lgr5-GFPhi ISCs or Lgr5-GFPlow progenitors by pooling two to five 71-week old male mice per HFD and HFD-control using Tri Reagent (Life Technologies) according to the manufacturer's instructions, except for a overnight isopropanol precipitation at $-20^{\circ} \mathrm{C}$. From the total RNA, poly $(A)^{+}$RNA was selected using Oligo(dT) ${ }_{25}$-Dynabeads (Life technologies) according to the manufacturer's protocol.

RNA-Seq Library Preparation. Strand-specific RNA-seq libraries were prepared using the dUTP-based, Illumina-compatible NEXTflex Directional RNA-Seq Kit (Bioo Scientific) according to the manufacturer's directions. All libraries were sequenced with an Illumina HiSeq 2000 sequencer.

Processing of RNA-seq reads and measuring expression level. Raw stranded reads (40 nt) were trimmed to remove adapter and bases with quality scores below 20 , and reads shorter than $35 \mathrm{nt}$ were excluded. High-quality reads were mapped to the mouse genome (mm10) with TopHat version 1.4 .1 (Trapnell et al., 2009), using known splice junctions from Ensembl Release 70 and allowing at most 2 mismatches. Genes were quantified with htseq-count (with the "intersect strict" mode) using Ensembl Release 70 gene models. Gene counts were normalized across all samples using estimateSizeFactors() from the DESeq R/Bioconductor package(Anders and Huber, 2010). Differential expression analysis was also performed between two samples of interest with DESeq. 


\section{qRT-PCR}

Cells were sorted into Tri Reagent (Life Technologies) and total RNA was isolated according to the manufacturer's instructions with following modification: the aqueous phase containing total RNA was purified using RNeasy plus kit (Qiagen). RNA was converted to CDNA with cDNA synthesis kit (Bio-Rad). qRT-PCR was performed with SYBR green master mix (Bio-Rad) on Bio-Rad iCycler RT-PCR detection system. For low cell numbers $(<1000)$, qRT-PCR was performed after sequence specific preamplification as described in single-cell gene expression analysis. Primers used are listed on Supplementary Table 1.

\section{Single cell RNA-Seq}

\section{Cell sorting}

FACS (Astrios) was used to sort one single cell into each well of a 96-well PCR plate containing $5 \mu \mathrm{l}$ of TCL buffer with $1 \% 2$-mercaptoethanol. The cells were stained for 7AAD (Life Technologies), CD45 (eBioscience), CD31- (eBioscience), Ter119 (eBioscience), $\mathrm{EpCAM}^{+}$(eBioscience). To enrich for specific IEC populations, cells were isolated from control or HFD Lgr5-GFP mice, stained with the antibodies mentioned above and gated for GFP-high (stem cells) and GFP-low (TAs). A population control of 200 cells was sorted into one well and a no-cell control was sorted into another well. After sorting, the plate was sealed tightly with a Microseal $\mathrm{F}$ and centrifuged at $800 \mathrm{~g}$ for $1 \mathrm{~min}$. The plate was immediately frozen on dry ice and kept at $-80^{\circ} \mathrm{C}$ until ready for the lysate cleanup.

Plate-based scRNA-seq 
Libraries were prepared using a modified SMART-Seq2 protocol as previously reported (Picelli et al., 2014). Briefly, RNA lysate cleanup was performed using RNAClean XP beads (Agencourt), followed by reverse transcription with Maxima Reverse Transcriptase (Life Technologies) and whole transcription amplification (WTA) with KAPA HotStart HIFI 2x ReadyMix (Kapa Biosystems) for 21 cycles. WTA products were purified with Ampure XP beads (Beckman Coulter), quantified with Qubit dsDNA HS Assay Kit (ThermoFisher), and assessed with a high sensitivity DNA chip (Agilent). RNA-seq libraries were constructed from purified WTA products using Nextera XT DNA Library Preparation Kit (Illumina). On each plate, the population and no-cell controls were processed using the same method as the single cells. The libraries were sequenced on an Illumina NextSeq 500.

\section{Computational analysis of scRNA-seq}

We profiled Lgr5-high ISCs sorted from control ( $n=192$ cells) and HFD ( $n=192$ cells) using a full length scRNA-seq method (Picelli et al., 2014). Each condition included two replicate 96-well plates from 2 different mice. Expression levels of gene loci were quantified using RNA-seq by Expectation Maximization (RSEM) (Li and Dewey, 2011). Raw reads were mapped to a mouse transcriptome index (mm10 UCSC build) using Bowtie 2 (Langmead and Salzberg, 2012), as required by RSEM in its default mode. On average, $90 \%$ of the reads mapped to the genome in every sample. and $55 \%$ of the reads mapped to the transcriptome. RSEM yielded an expression matrix (genes $\mathrm{x}$ samples) of inferred gene counts, which was converted to TPM (transcripts per million) values and then log-transformed after the addition of 1 to avoid zeros. After filtering cells with low QC metrics (< 400,000 mapped reads, transcriptomic mapping rate $<35 \%$ and 
$<1500$ genes detected), we selected 171 control cells and 144 HFD cells for further analysis.

We identified 379 highly variable genes using Seurat's MeanVarPlot function. H2-Ab1, a key component of MHC-II complex, was among the top 5 differentially expressed genes with > 3-fold higher expression in control compared to HFD ISCs as assessed using MAST test.(Finak et al., 2015) A Gene Ontology analysis of these genes against a background of genes matched in average expression levels showed an enrichment of terms consistent with intestinal biology such as arachidonic acid metabolic process (GO:0019369), intestinal absorption (GO:0050892), as well as immune response, such as "antigen processing and presentation of exogenous peptide antigen" (GO:0002478) and "defense response to Gram-negative (GO:0050829), and Gram-positive (GO:0050830) bacterium". We performed principal component analysis (PCA) on the data based on the variable genes, and embedded 10 statistically significant PCs identified using a permutation test (Shekhar et al., 2016) on a 2D map using tdistributed stochastic neighbor embedding (tSNE).

\section{DQ-Ovalbumin Assay}

Lgr5-GFP ${ }^{\text {hi }}$ intestinal stem cells were plated in Matrigel and crypt media for one day.

The following day, the crypt media was replaced with crypt media containing $20 \mu \mathrm{g} / \mathrm{mL}$ DQ-ovalbumin (Invitrogen) and cells were incubated at 4C and 37C for 24 hours. Cells were harvested by washing three times in PBS, removing the Matrigel using Cell 
Recovery Solution (Corning) and filtered through a $40 \mu \mathrm{m}$ mesh. Mean fluorescent intensity was analyzed using CellSimple (Cell Signaling).

\section{Orthotopic transplantation}

$A p c^{L / L}$; Lgr5-EGFP-IRES-CreERT2 mice were injected with two doses of tamoxifen I.P. Four days later, Apc-null Lgr5-GFPhi ISCs and Lgr5-GFPlow progenitors were sorted by flow cytometry, as described above. For primary cell transplantations, 10,000 Apc-null Lgr5-GFP ${ }^{\text {hi }}, \mathrm{MHC}^{\text {hi }}$ and MHClow ISCs were resuspended into $90 \%$ crypt culture media (as described) and $10 \%$ Matrigel $^{\mathrm{TM}}$, and then transplanted into the colonic lamina propria of C57BL/6 recipient mice as previously described (Beyaz et al., 2016; Roper et al., 2017). Mice then underwent colonoscopy eight weeks later to assess tumor formation. Colonoscopy videos and images were saved for offline analysis. Following sacrifice, the distal colons were excised and fixed in $10 \%$ formalin, then examined by hematoxylin and eosin section to identify adenomas. Histology images were reviewed by gastrointestinal pathologists who were blinded to treatment groups.

\section{Taxonomic Microbiota Analysis.}

Frozen fecal samples were processed for DNA isolation using the Qiagen PowerSoil kit according to the manufacturer's instructions. $1 \mathrm{ng}$ of purified fecal DNA was used for PCR amplification. Amplicons spanning the variable region 1/2 (V1/2) of the 16S rRNA gene were generated by using the following barcoded primers: Fwd 5'XXXXXXXXAGAGTTTGATCCTGGCTCAG-3', Rev 5'-TGCTGCCTCCCGTAGGAGT-3', where $X$ represents a barcode base. The reactions were subsequently pooled and cleaned, and the PCR products were then sequenced on an Illumina MiSeq with $500 \mathrm{bp}$ 
paired-end reads. The reads were then processed using the QIIME (Quantitative Insights Into Microbial Ecology, http://www.qiime.org) analysis pipeline. Rarefaction was used to exclude samples with insufficient count of reads per sample. Sequences sharing $97 \%$ nucleotide sequence identity in the $16 \mathrm{~S}$ region were binned into operational taxonomic units (97\% ID OTUs). For beta-diversity, unweighted UniFrac measurements were plotted according to the two principal coordinates based on $>10,000$ reads per sample.

\section{Figure Legends}

Figure 1 | High Fat Diet reduces MHC-II expression on Lgr5-GFP ${ }^{\text {hi }}$ intestinal stem cells (ISCs).

A. A heat map of downregulated genes assessed by bulk RNA-seq in Lgr5-GFPhi intestinal stem cells (Lgr5+ ISCs) isolated from long-term high fat diet (HFD)-fed mice compared to control mice $(n=2)$.

B. Violin plots demonstrating MHC-II expression in single ISCs isolated from control ( $n=171$ cells, 2 independent experiments) or HFD mice by single cell RNA-seq (scRNA-seq) ( $\mathrm{n}=144$ cells, 2 independent experiments).

C. Control (blue) and HFD (red) Lgr5+ ISCs ranked according to their expression of MHC II pathway genes (y-axis). Dashed lines correspond to y-intercepets of -0.2 and 0.75 , which are the $25^{\text {th }}$ and $75^{\text {th }}$ percentile of scores in HFD cells, used to define MHC2 low (score < -0.2) and MHC2 high (score > 0.75) HFD cells. In contrast, these values correspond to $1^{\text {st }}$ and $35^{\text {th }}$ percentile of scores in control cells. 
D. Heatmap showing differentially expressed (DE) genes (rows) between MHC2 low and MHC2 high HFD ISCs as defined in panel C.

E. Single-molecule in situ hybridization of MHC-II (H2-Ab1) in control and HFD mice $(n=5)$.

F, G. Mean fluorescence intensity (MFI) of MHC-II in Epcam+ cells from crypts of control and HFD mice (F, $n=10$ mice). Representative flow cytometry histogram plots of MHC-II expression in Epcam+ cells (G).

H. Relative expression of MHC-II (H2-Ab1) in Epcam+ cells isolated from crypts of control and HFD mice ( $n=10$ mice).

I, J. Mean fluorescence intensity (MFI) of MHC-II in Lgr5+ ISCs from crypts of control and HFD mice (I, $n=10$ mice). Representative flow cytometry histogram plots of MHC-II expression in Epcam+ cells $(\mathbf{J})$.

K. Relative expression of MHC-II (H2-Ab1) in Lgr5+ ISCs isolated from crypts of control and HFD mice ( $n=10$ mice).

L, M. Frequency of MHC-II+ and MHC-II- ISCs in control and HFD mice by flow cytometry (L, $n=4$, mean $\square \pm \square$ s.d). Representative flow cytometry plots of MHC-II in control and HFD ISCs (M).

Unless otherwise indicated, data are mean $\square \pm \square$ s.e.m. from $n$ independent experiments; ${ }^{\star} P \square<\square 0.05,{ }^{* *} P \square<\square 0.01,{ }^{* * *} P \square<\square 0.001$ (Student's $t$-tests). Scale bars, $50 \square \mu \mathrm{m}$ (E) and $20 \square \mu \mathrm{m}(\mathbf{E}$, inset).

Figure 2 | Intestinal microbiome regulates MHC-II expression in ISCs 
A. In situ hybridization for $\mathrm{H} 2-A b 1$ in control, HFD, and antibiotic-treated mice in proximal small intestine $(n=5)$.

B. Relative expression of MHC-II in Lgr5+ ISCs from control, HFD, and antibiotictreated mice ( $n=5$, mean $\square \pm \square$ s.d).

C. Single-molecule in situ hybridization of MHC-II (H2-Ab1) in specific pathogenfree (SPF) and germ-free mice ( $n=5$ mice).

D. Relative expression of MHC-II $(H 2-A b 1)$ in Lgr5+ ISCs from SPF and germfree mice $(n=5)$.

E, F. Mean fluorescence intensity (MFI) of MHC-II in Lgr5+ ISCs from SPF and germ-free mice (E, $n=5)$. Representative flow cytometry histogram plots of MHC-II expression in Lgr5+ ISCs (F).

G. Volcano plot demonstrating significantly enriched and depleted microbial species in HFD versus control mice $(n=5)$.

H. Relative abundance of Helicobacter sp. in mice housed in clean room and dirty room $(n=5)$.

I. Volcano plot demonstrating significantly enriched and depleted microbial species in mice housed in dirty room versus clean room $(n=6)$.

J. Relative expression of MHC-II $(H 2-A b 1)$ in Epcam+ cells isolated from crypts of mice housed in clean room or dirty room $(n=7)$.

K. Relative abundance of Helicobacter sp. in mice housed either in clean room $(n=10)$, dirty room $(n=3)$, or after co-housing clean mice $(n=10)$ with dirty mice $(n=4)$ in dirty room. 
L. Relative expression of MHC-II (H2-Ab1) in Epcam+ cells isolated from crypts of mice housed either in clean room, dirty room, or after co-housing clean mice with dirty mice in dirty room ( $n=5$, ANOVA).

M, N. Mean fluorescence intensity (MFI) of MHC-II in Epcam+ cells isolated from crypts of mice housed either in clean room, dirty room, or after co-housing clean mice with dirty mice in dirty room ( $\mathbf{M}, n=5$, ANOVA). Representative flow cytometry histogram plots of MHC-II expression in Lgr5+ ISCs $(\mathbf{N})$.

Unless otherwise indicated, data are mean $\square \pm \square$ s.d. from $n$ independent experiments; ${ }^{*} P \square<\square 0.05,{ }^{* *} P \square<\square 0.01,{ }^{* * *} P \square<\square 0.001$ (Student's $t$-tests).

Figure 3 | PRR and IFN $\gamma$ signaling regulate MHC-II expression in ISCs.

A. Relative expression of pattern recognition receptors (PRR) in control and HFD ISCs $(n=3)$.

B. Mean fluorescence intensity (MFI) of MHC-II in ISCs from vehicle- and TLR2/NOD2 agonist CL429- treated control and HFD mice ( $n=6$ mice).

C. A heat map of expression levels of IFN $\gamma$-induced genes between HFD and control ISCs by bulk RNA-seq ( $n=2)$.

D. Violin plots demonstrating the expression levels of IFN $\gamma$-induced genes in control and HFD ISCs by scRNA-seq.

E. IFN $\gamma$ levels in the intestines of control and HFD mice as measured by ELISA $(n=10)$.

F. In situ hybridization for $H 2-A b 1$ in vehicle- and CYT387-treated mice in small intestine $(n=3)$. 
G. Relative expression of MHC-II (H2-Ab1) in Lgr5+ ISCs from vehicle- and Jak/Stat inhibitor CYT387-treated mice $(n=7)$.

H, I. Mean fluorescence intensity (MFI) of MHC-II in Lgr5+ ISCs from vehicleand CYT387-treated mice $(\mathbf{H}, n=5)$. Representative flow cytometry histogram plots of MHC-II expression in Lgr5+ ISCs (I).

J. Relative expression of MHC-II (H2-Ab1) in intestinal organoids-treated with or without CYT387 and/or IFN $\gamma(n=5)$.

K. Relative expression of MHC-II (H2-Ab1) in Epcam+ cells isolated from crypts of control or IFNGR KO $(n=5)$.

Unless otherwise indicated, data are mean $\square \pm \square$ s.e.m from $n$ independent experiments; ${ }^{\star} P \square<\square 0.05,{ }^{* *} P \square<\square 0.01,{ }^{* * *} P \square<\square 0.001$ (Student's $t$-tests).

Figure 4 | Loss of MHC-II in premalignant ISCs increases tumor initiation.

A, B. Organoid-initiating capacity of control and HFD MHC-II+ and MHC-II- Apcnull ISCs at day 5 (control MHC-II+, $n=3$, control MHC-II-, $n=3$, HFD MHC-II+, $n=4$, HFD MHC-II-, $n=4)$. Representative images of HFD MHC-II+ and MHC-II- Apc-null organoids at day $5(\mathbf{B})$.

C. Tumor initiation rate of orthotopically transplanted MHC-II+ and MHC-II- Apcnull ISCs from HFD mice into immunocompetent syngeneic hosts $(n=8)$.

D. Tumor initiation rate of orthotopically transplanted MHC-II+ and MHC-II- Apcnull ISCs from HFD mice into immunodeficient (Rag2 KO) hosts $(n=8)$.

E, F. Tumor size index in distal colon of mice that received tamoxifen through endoscopy guided tamoxifen injection to induce tumor formation upon loss of APC (E, 
n=6, MHC-II WT APC KO: villin-Cre-ERt2 APC L/L, MHC-II L/+, MHC-II iKO APC KO: villin-Cre-ERt2 APC L/L, MHC-II L/L). Representative optical colonoscopy images of tumors $(\mathbf{F})$.

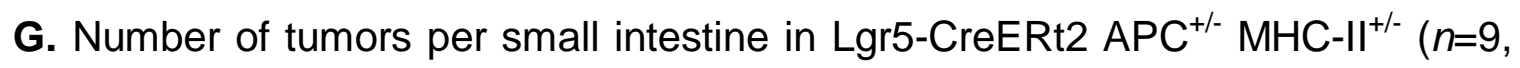
MHC-II WT APC-het) and Lgr5-GFP+ $\mathrm{APC}^{+--} \mathrm{MHC}^{-/-}(n=9, \mathrm{MHC}-\mathrm{II}$ iKO APC KO) mice 5 months post tamoxifen injection.

Unless otherwise indicated, data are mean $\square \pm \square$ s.e.m from $n$ independent experiments; ${ }^{*} P \square<\square 0.05,{ }^{* *} P \square<\square 0.01,{ }^{* *} P \square<\square 0.001$ (Student's $t$-tests).

\section{Supplementary Figure 1 | Characterization of the MHC-II+ and MHC-II- ISCs.}

A. Relative expression of immunomodulatory genes in control and HFD Lgr5$\operatorname{GFP}^{\text {hi }}$ ISCs $(n=5)$.

B. $t$-Distributed stochastic neighbour embedding (tSNE) analysis of single ISCs isolated from control ( $n=171$ cells, 2 independent experiments) or HFD mice ( $n=144$ cells, 2 independent experiments).

C. tSNE analysis of single cells using MHC-Il pathway signature genes

D. Relative expression of MHC-II in control and HFD MHC-II+ and MHC-II- ISCs $(n=5)$

E. Relative expression of signature PPAR-d target (Hmgcs2), stem cell marker (Lgr5), PPAR-d-dependent B-catenin target (Jag1) and IFNGR genes in control and HFD MHC-II+ and MHC-II- ISCs $(n=5)$.

F. Organoid-initiating capacity of MHC-II+ and MHC-II- Lgr5-GFPhi ISCs from HFD mice $(n=5)$. 
G. Mean fluorescence intensity (MFI) of ISCs pulsed with either vehicle or DQOvalbumin for 8 hours at $4 \mathrm{C}$ and $37 \mathrm{C}(n=5,3$ technical replicates per experiment).

H. Weight of mice used for diet-induced obesity (left: Control and HFD, $n=15$ ) and leptin receptor deficiency models of obesity (right, Control and $\mathrm{db} / \mathrm{db}, n=7$ ) in the study.

I, J. Frequency of MHC-II+ and MHC-II- ISCs in vehicle- and PPAR-delta agonist GW501516-treated mice by flow cytometry (I, $n=3)$. Representative flow cytometry plots of MHC-II in vehicle- and PPAR-delta agonist GW501516-treated ISCs (J).

K, L. Frequency of MHC-II+ and MHC-II- ISCs in lean $\mathrm{db} /+$ and obese $\mathrm{db} / \mathrm{db}$ mice $(\mathbf{K}, n=3)$. Representative flow cytometry plots of $\mathrm{MHC}-\mathrm{II}+$ and MHC-II- ISCs (I, $n=3)$.

Unless otherwise indicated, data are mean $\square \pm \square$ s.d. from $n$ independent experiments; ${ }^{\star} P \square<\square 0.05,{ }^{* *} P \square<\square 0.01,{ }^{* * *} P \square<\square 0.001$ (Student's $t$-tests).

Supplementary Figure 2 | Intestine-specific deletion of MHC-II does not

\section{significantly alter intestinal physiology.}

A-E, Intestinal weight (a, $n=5)$, length (b, $n=5)$, crypt $(\mathbf{c}, n=5)$, and villi length (d, $n=5)$ of MHC-II wild type (WT) and MHC-II L/L; Villin-CreERT2 (vil-iKO) mice one month after tamoxifen injection. Representative H\&Es of WT and vil-iKO small intestine (E).

$\mathbf{F}, \mathbf{G}$, In situ hybridization for $H 2-A b 1$ in the intestine $(\mathbf{F})$ and representative images of intestinal crypts $(\mathbf{G})$ in WT and vil-iKO mice $(n=3)$.

H. Frequencies of MHC-II+ and MHC-II- Lgr5-GFP ${ }^{\text {hi }}$ ISCs in WT and Lgr5-GFP MHC-II-deleted (Lgr5-iKO) by flow cytometry $(n=5)$. 
I. Lineage tracing of LacZ+ cells in the intestinal crypt after deletion of MHC-II in ISCs (left). Representative images of LacZ immunostain in WT and Lgr5-iKO small intestine (right).

Unless otherwise indicated, data are mean $\square \pm \square$ s.d. from $n$ independent experiments; ${ }^{*} P \square<\square 0.05,{ }^{* *} P \square<\square 0.01,{ }^{* *} P \square<\square 0.001$ (Student's $t$-tests). Scale bars, $100 \square \mu \mathrm{m}(\mathbf{E}), 50 \square \mu \mathrm{m}(\mathbf{F}, \mathbf{I}, \mathbf{K})$ and $20 \square \mu \mathrm{m}(\mathbf{G}, \mathbf{I}$, (insets)).

Supplementary Figure 3 | Intestine-specific deletion of MHC-II does not alter the organoid forming capacity of ISCs.

A, B. Number of bromodeoxyuridine (BrdU)+ crypt base columnar cells after a 4hour pulse in MHC-IIL/ Villin-CreERT2 (vil-iKO) mice after tamoxifen administration (WT: $n=3$, vil-iKO: $n=4$ ). Representative images of BrdU immunostain in proximal small intestinal crypts (B).

C-E Organoid-initiating capacity of WT and vil-iKO crypts (C, $n=5)$. Number of secondary organoids per dissociated crypt-derived primary organoid (D, $n=5$ ). Representative images of day-5 WT and vil-iKO primary organoids (E).

F. Relative expression of MHC-II in dissociated WT and vil-iKO primary organoids at day $5(n=5)$.

G, H. Organoid-initiating capacity of ISCs from WT and MHC-II ${ }^{L / L}$; Lgr5-EGFPIRES-CreERT2 (Lgr5-iKO) mice with and without Paneth cells (P) from WT mice $(n=5)$. Representative images of organoids derived from WT and Lgr5-iKO ISCs co-cultured with WT Paneth cells five days after seeding $(\mathbf{H})$. 
Unless otherwise indicated, data are mean $\square \pm \square$ s.d. from $n$ independent experiments;

${ }^{\star} P \square<\square 0.05,{ }^{* *} P \square<\square 0.01,{ }^{* *} P \square<\square 0.001$ (Student's $t$-tests). Scale bars, $100 \square \mu \mathrm{m}$ (E, H) and $20 \square \mu \mathrm{m}(\mathbf{B})$.

\section{Supplementary Figure 4 | Microbial regulation of MHC-II expression in ISCs.}

A. Chao1 index of microbial diversity in Control, HFD, and mice treated with antibiotics for 3 months $(n=5)$.

B. Principle component analysis of microbial diversity in Control, HFD, and antibiotic-treated mice $(n=5)$.

C, D. Frequencies of MHC-II+ and MHC-II- ISCs in specific-pathogen free (SPF) and germ-free mice by flow cytometry $(n=3)$. Representative flow cytometry plots of MHC-II in SPF and germ-free ISCs (D).

E. Principle component analysis of microbial diversity in mice housed in clean room or dirty room $(n=6)$.

F. Relative abundance of Odoribacter sp. in mice housed in clean room or dirty room $(n=6)$.

G. Principle component analysis of microbial diversity at baseline level before cohousing experiment (left) and 10 days after co-housing clean mice with dirty mice in the dirty room.

Unless otherwise indicated, data are mean $\square \pm \square$ s.d. from $n$ independent experiments; ${ }^{\star} P \square<\square 0.05,{ }^{* *} P \square<\square 0.01,{ }^{* * *} P \square<\square 0.001$ (Student's $t$-tests). 


\section{Supplementary Figure 5 | Regulation of MHC-II expression in ISCs by PRR}

\section{and Jak/Stat signaling.}

A, B. In situ hybridization for $\mathrm{H} 2-\mathrm{Ab} 1$ in control and HFD mice treated with vehicle- and CL429-treated mice in proximal small intestine (A, $n=3)$. Representative histogram plots of MHC-II in ISCs in control and HFD mice treated with vehicle and CL429 (B, $n=3)$.

C. In situ hybridization for H2-Ab1 in WT and Myd88 KO mice ( $n=4)$.

D. Relative expression of Myd88 and MHC-II genes (H2-Aa and H2-Ab1) in the intestine from WT and Myd88 KO mice $(n=5)$.

E. Relative expression of MHC-II (H2-Ab1) in MHC-II+ and MHC-II- ISCs isolated from HFD mice with or without IFNg stimulation $(n=4)$.

F. Violin plots demonstrating the levels of IFNg-induced genes excluding MHC-II pathway genes in control and HFD ISCs by scRNA-seq.

G. Violin plots demonstrating the levels of STAT3 in control and HFD ISCs by scRNA-seq.

H, I. Frequencies of MHC-II+ and MHC-II- ISCs in vehicle- and Jak/Stat inhibitor CYT387-treated mice $(n=3)$. Representative flow cytometry plots of MHC-II in vehicleand CYT387-treated ISCs (I).

Unless otherwise indicated, data are mean $\square \pm \llbracket$ s.d. from $n$ independent experiments; ${ }^{*} P \square<\square 0.05,{ }^{* *} P \square<\square 0.01,{ }^{* * *} P \square<\square 0.001$ (Student's $t$-tests). Scale bars, $50 \square \mu \mathrm{m}$ (A, C) and $20 \square \mu \mathrm{m}$ (insets, A). 


\section{Supplementary Figure 6 | The effect of HFD on intestinal immune cells}

A. Frequency of CD45+ cells in intestinal crypts isolated from control or HFD mice $(n=7)$.

B. Frequency of CD3+ $T$ cells, CD8+ $T$ cells and CD4+ $T$ cells among CD45+ cells in intestinal crypts isolated from control or HFD mice $(n=5)$.

C-H. Numbers of CD8+ cells (C, crypt; D, villus) and CD4+ cells (E, crypt; $\mathbf{F}$, villus) in the intestines of control or HFD mice. Representative images of CD4 and CD8 immunostaining in control or HFD intestines (G, $\mathbf{H})$.

\section{Supplementary Figure 7 | The effect of ISC MHC-II expression on tumor} formation.

A, B. Fraction of $H 2-A b 1+$ tumor areas per total tumor area by single-molecule in situ hybridization in control and HFD Lgr5+ Apc-null mice 14 days post tamoxifen injection (Control, $n=6$; HFD, $n=5$ ). Representative images of $\mathrm{H} 2$-Ab1 in situ hybridization in control and HFD tumors from Lgr5+ Apc-null mice (B).

C. Relative expression of MHC-II (H2-Ab1) in Lgr5+ Apc-null pre-malignant ISCs isolated from control or HFD mice 3 days post tamoxifen injection $(n=5)$.

D. Mean fluorescence intensity (MFI) of MHC-II in Lgr5+ Apc-null pre-malignant ISCs isolated from control or HFD mice 3 days post tamoxifen injection ( $n=6)$. 
E, F. Frequency of MHC-II+ and MHC-II- Lgr5-GFP ${ }^{\text {hi }} A p c$-null ISCs in control and HFD mice by flow cytometry ( $E, n=5)$. Representative flow cytometry plots of MHC-II in HFD Apc-null ISCs (F).

G-I. Characterization of orthotopically transplanted MHC-II+ and MHC-II- Apc-null ISCs-derived tumors three months after transplantation into immunocompetent syngeneic hosts. Optical colonoscopy images $(\mathbf{G})$, Hematoxylin and eosin $(\mathbf{H})$ and betacatenin immunostain (I) of tumors.

J-L. Characterization of orthotopically transplanted MHC-II+ and MHC-II- Apcnull ISCs-derived tumors three months after transplantation into immunodeficient Rag2KO hosts. Optical colonoscopy images (J), H\&E (K) and beta-catenin immunostain (L) of tumors.

M-O. Tumors in Lgr5-Cre+ $\mathrm{APC}^{+-} \mathrm{MHC}^{+-}(\mathrm{MHC}-\mathrm{Il} \mathrm{WT})$ and Lgr5-Cre+ $\mathrm{APC}^{+/-}$ $\mathrm{MHC}^{-/}$(MHC-II iKO) mice. Representative images of tumors from Lgr5-Cre+ $\mathrm{APC}^{+/-}$ $\mathrm{MHC}^{+/-}\left(\mathbf{M}\right.$, left) and Lgr5-Cre+ $\mathrm{APC}^{+/-} \mathrm{MHC}^{-/-}(\mathbf{M}$, right) mice. Representative H\&E (N) and beta-catenin immunostain (0) images from tumors in Lgr5-GFP+ $\mathrm{APC}^{+/-} \mathrm{MHC}^{+-}$and Lgr5-GFP+ $\mathrm{APC}^{+-} \mathrm{MHC}^{-/-}$mice.

P-R. Numbers of CD3+ T cells per tumor area $(\mathbf{P})$. Representative H\&E $(\mathbf{N})$ and CD3 immunostain $(\mathbf{0})$ images from tumors.

\section{Method References:}

Anders, S., and Huber, W. (2010). Differential expression analysis for sequence count data. Genome biology 11, R106. 
Beyaz, S., Mana, M.D., Roper, J., Kedrin, D., Saadatpour, A., Hong, S.J., Bauer-Rowe, K.E., Xifaras, M.E., Akkad, A., Arias, E., et al. (2016). High-fat diet enhances stemness and tumorigenicity of intestinal progenitors. Nature 531, 53-58.

Colnot, S., Niwa-Kawakita, M., Hamard, G., Godard, C., Le Plenier, S., Houbron, C., Romagnolo, B., Berrebi, D., Giovannini, M., and Perret, C. (2004). Colorectal cancers in a new mouse model of familial adenomatous polyposis: influence of genetic and environmental modifiers. Laboratory investigation; a journal of technical methods and pathology 84, 1619-1630.

Finak, G., McDavid, A., Yajima, M., Deng, J., Gersuk, V., Shalek, A.K., Slichter, C.K., Miller, H.W., McElrath, M.J., Prlic, M., et al. (2015). MAST: a flexible statistical framework for assessing transcriptional changes and characterizing heterogeneity in single-cell RNA sequencing data. Genome Biol 16, 278.

Roper, J., Tammela, T., Cetinbas, N.M., Akkad, A., Roghanian, A., Rickelt, S., Almeqdadi, M., Wu, K., Oberli, M.A., Sanchez-Rivera, F.J., et al. (2017). In vivo genome editing and organoid transplantation models of colorectal cancer and metastasis. Nat Biotechnol 35, 569-576.

Sato, T., Vries, R.G., Snippert, H.J., van de Wetering, M., Barker, N., Stange, D.E., van Es, J.H., Abo, A., Kujala, P., Peters, P.J., et al. (2009). Single Lgr5 stem cells build crypt-villus structures in vitro without a mesenchymal niche. Nature.

Trapnell, C., Pachter, L., and Salzberg, S.L. (2009). TopHat: discovering splice junctions with RNA-Seq. Bioinformatics 25, 1105-1111.

Yilmaz, O.H., Katajisto, P., Lamming, D.W., Gultekin, Y., Bauer-Rowe, K.E., Sengupta, S., Birsoy, K., Dursun, A., Yilmaz, V.0., Selig, M., et al. (2012). mT0RC1 in the Paneth cell niche couples intestinal stem-cell function to calorie intake. Nature 486, 490-495. 


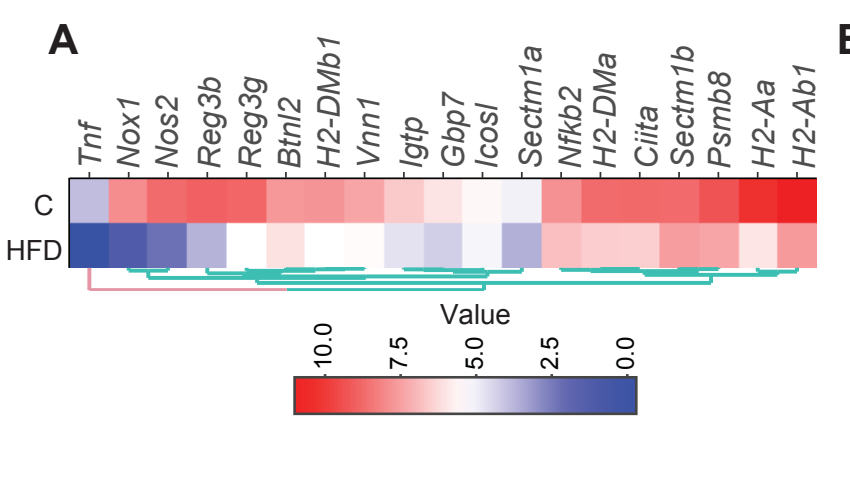

B

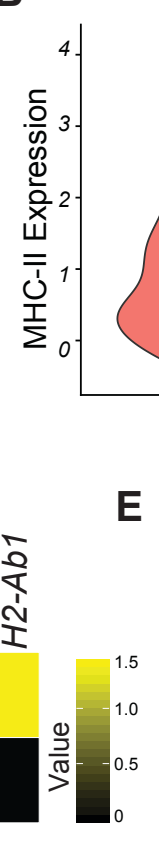

C

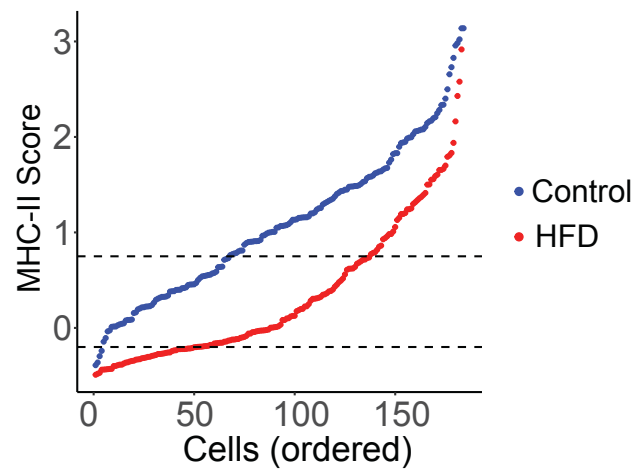

D

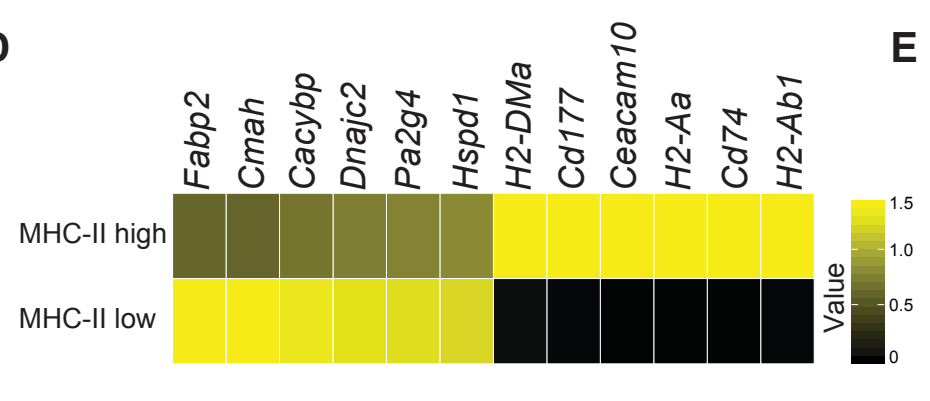

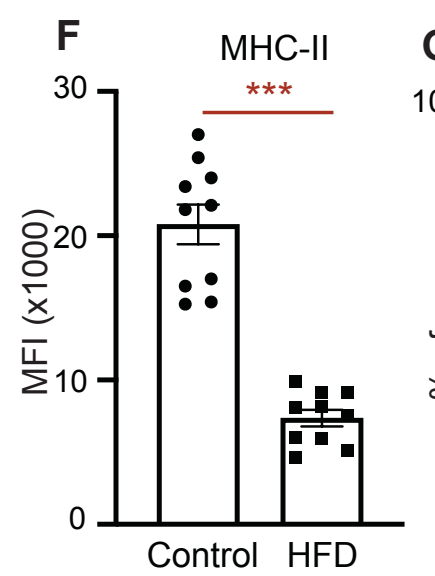

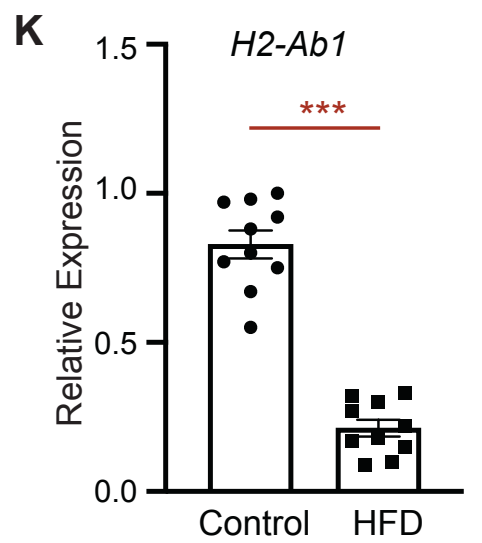

H

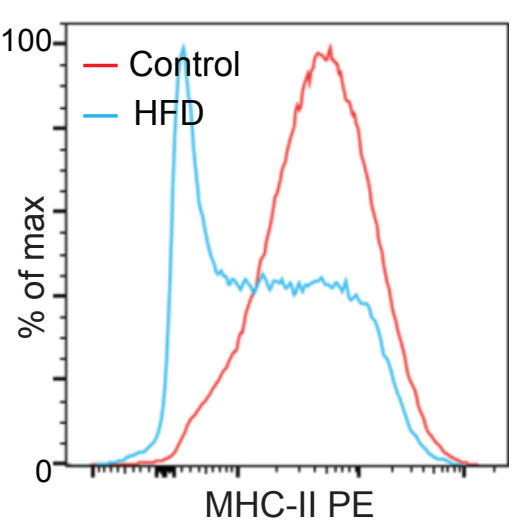

L

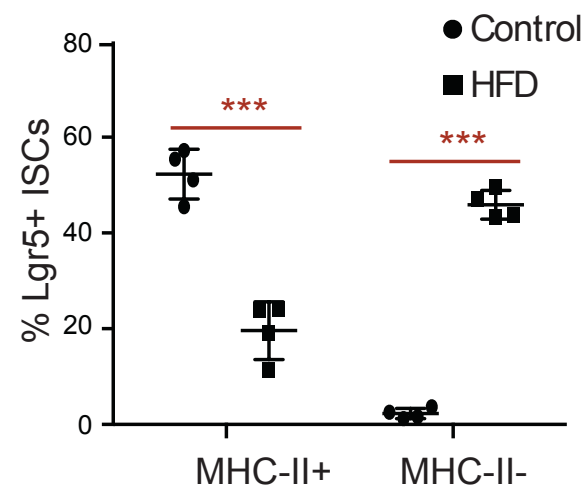

M

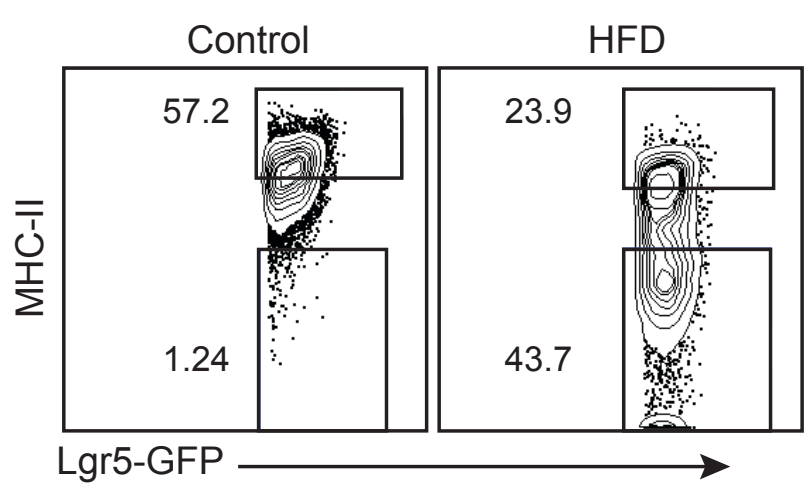

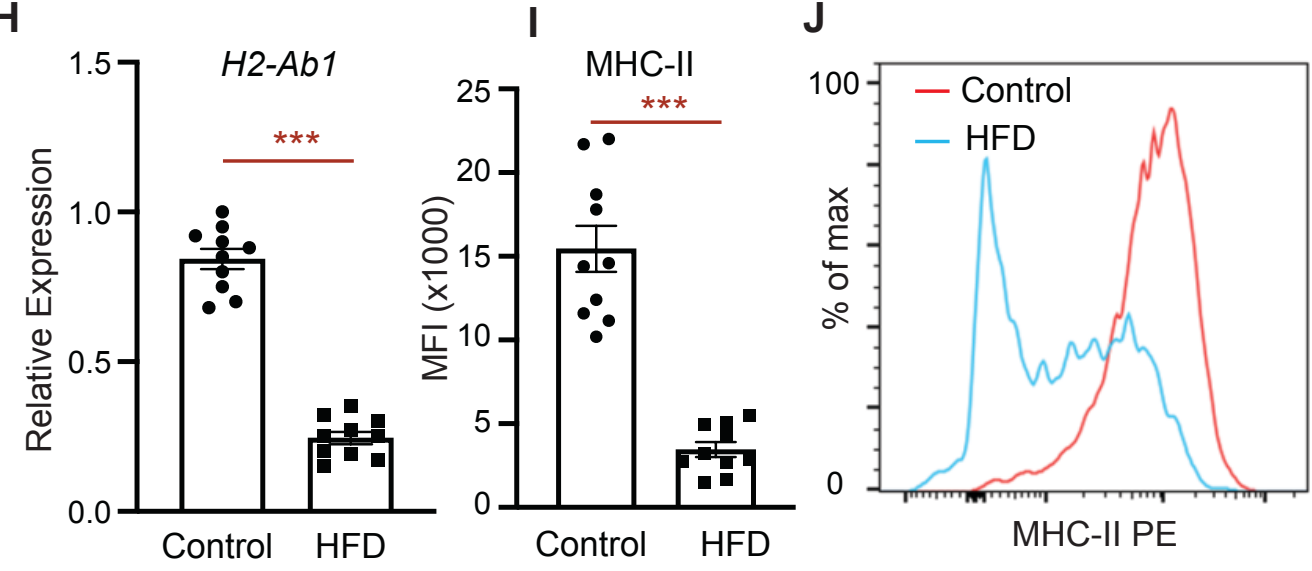




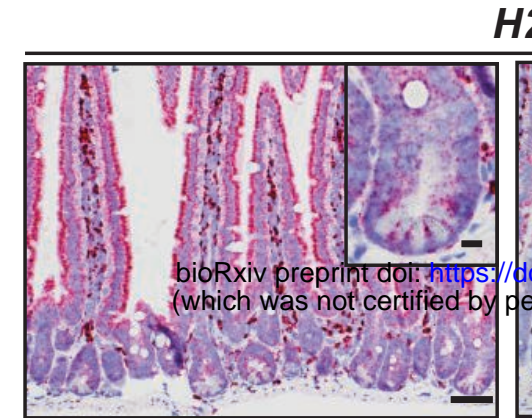

Control

C

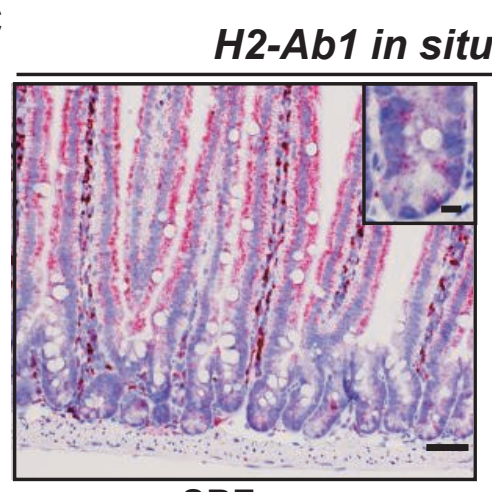

$\mathbf{F}$

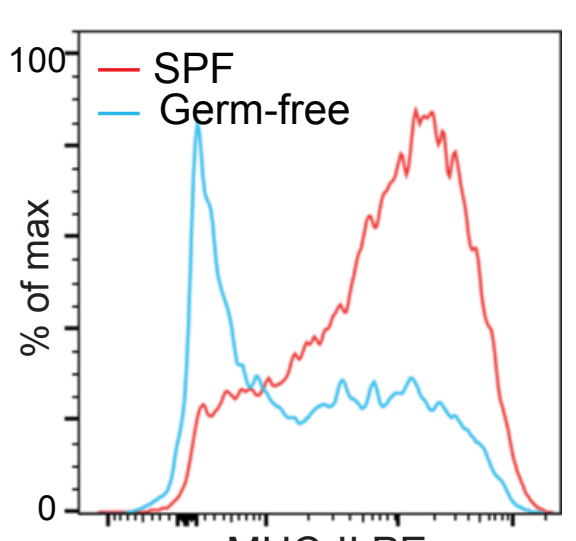

MHC-II PE
H2-Ab1 in situ hybridization

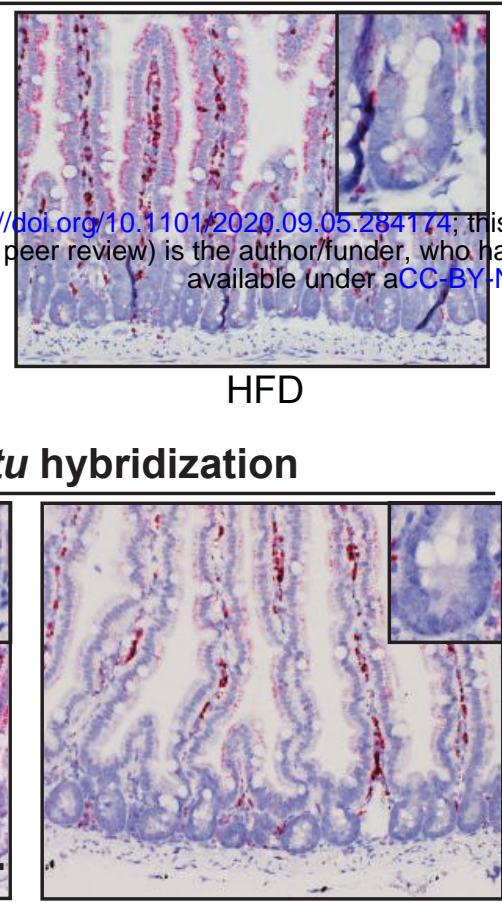

G

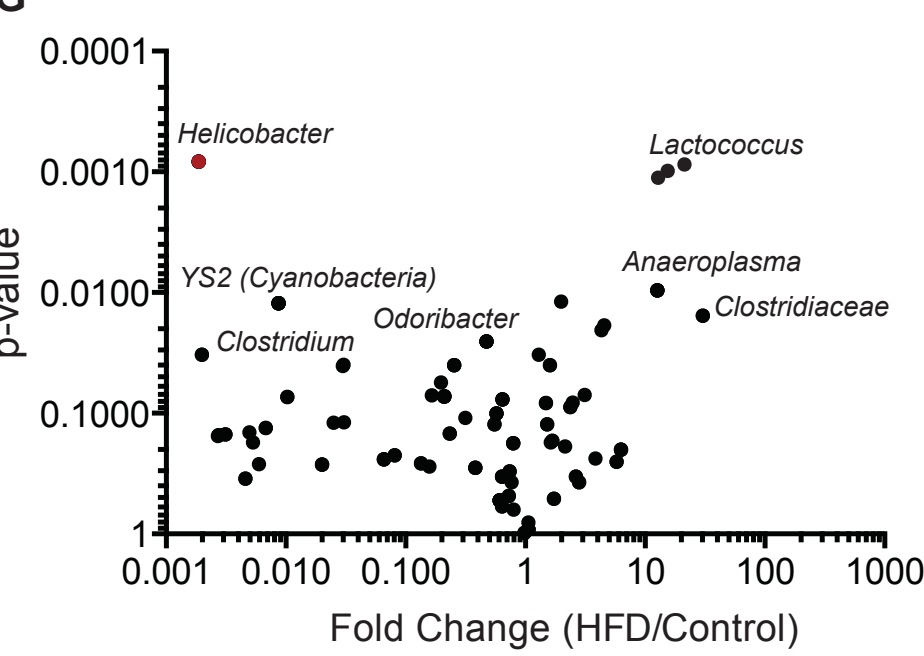

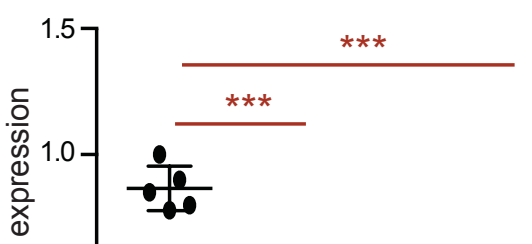

D
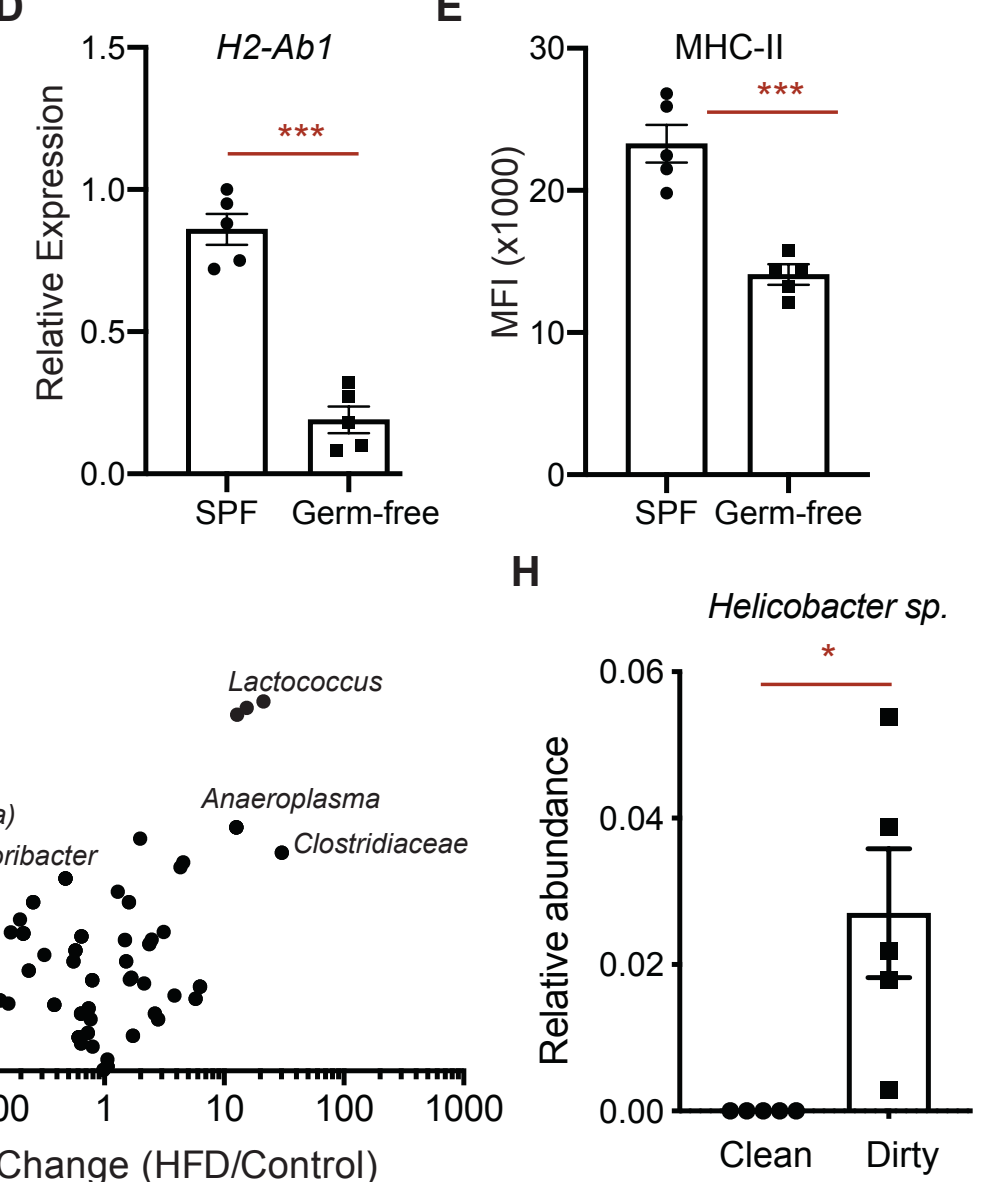

H Helicobacter $s p$.
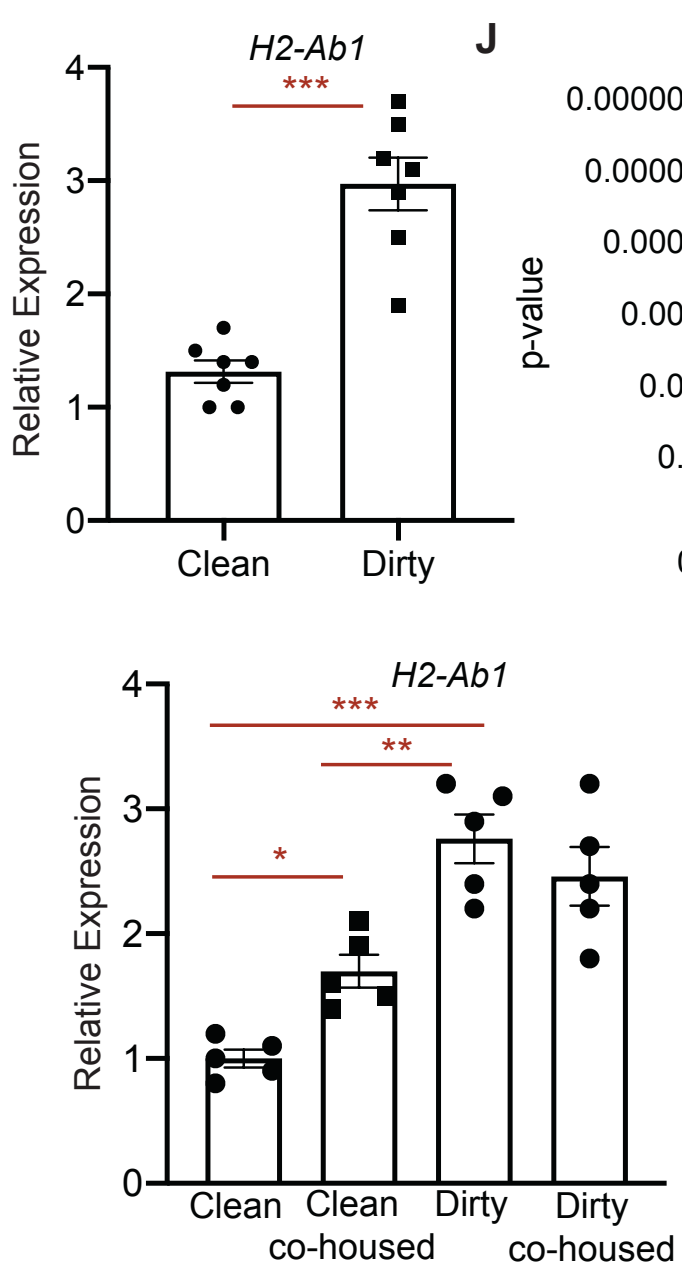

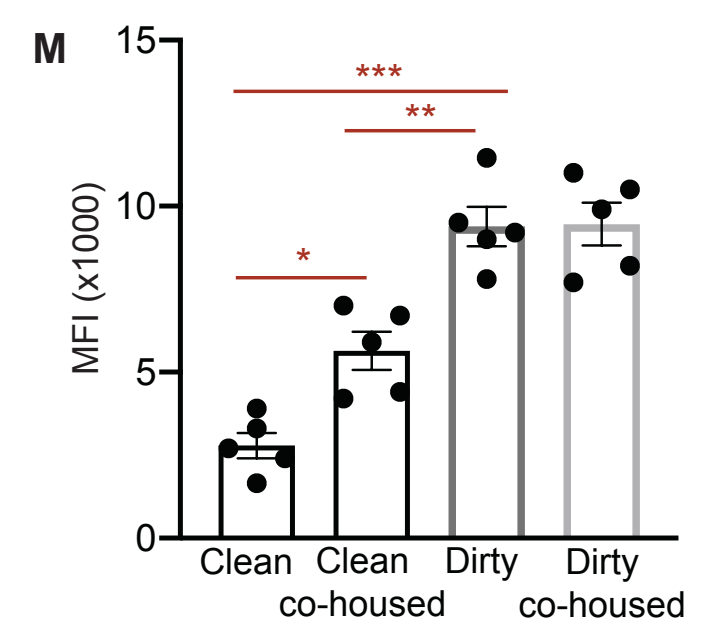

K

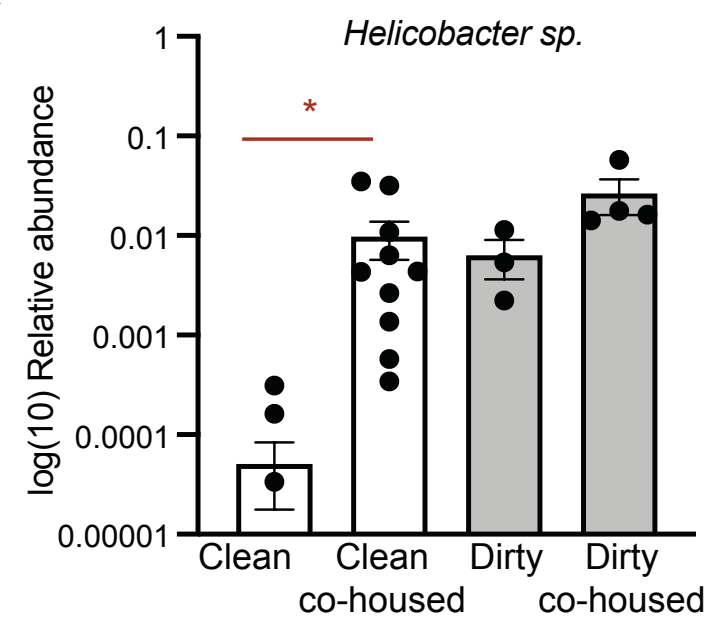

$\mathbf{N}$

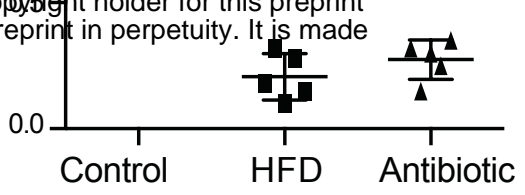

Dirty co-housed Dirty Clean co-housed 
Figure 3

A

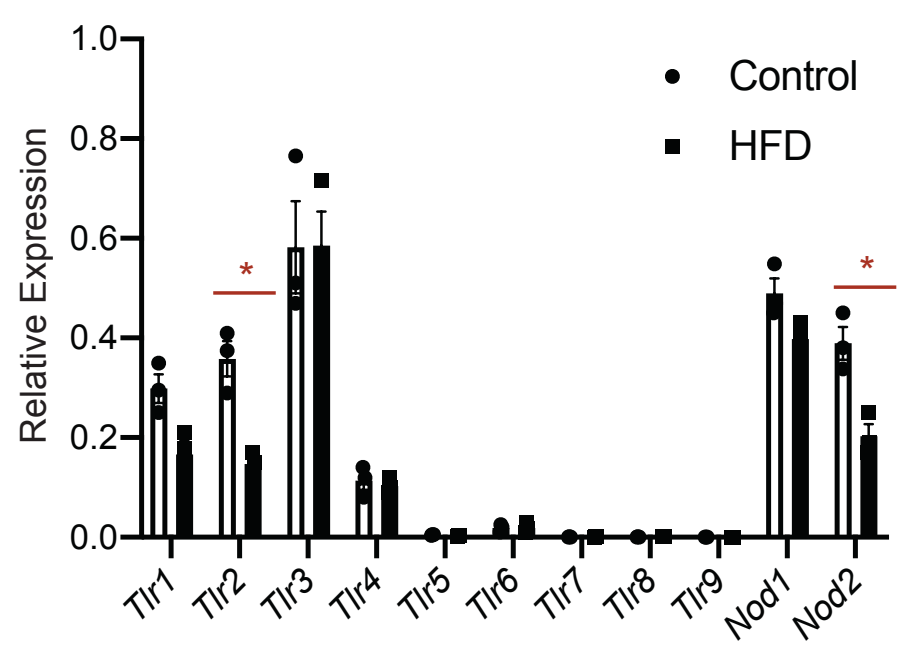

B

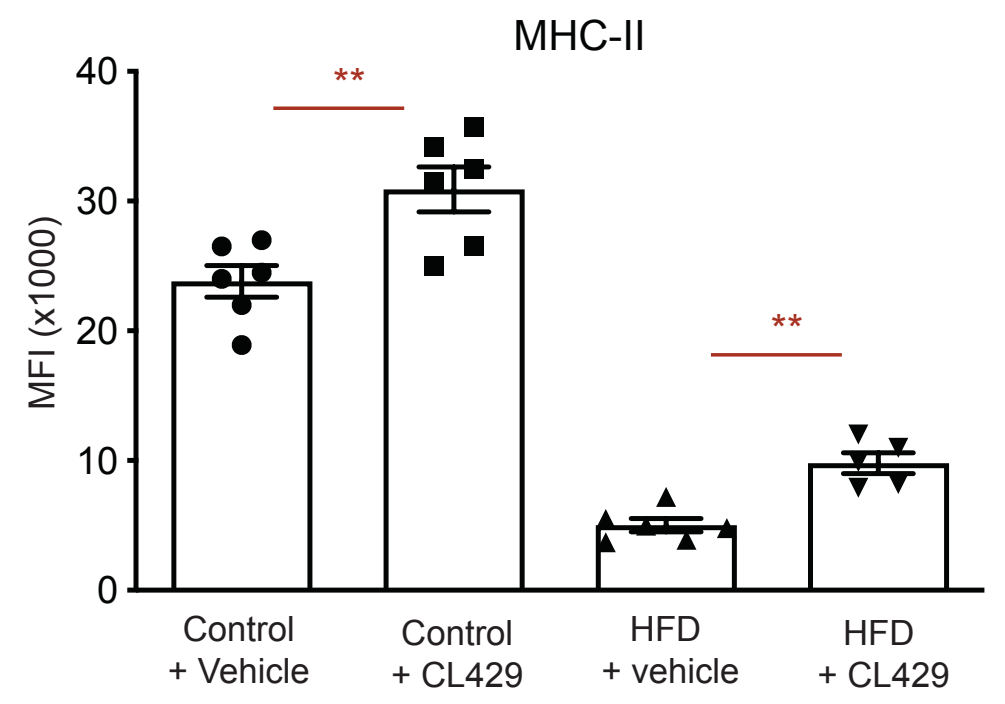

C

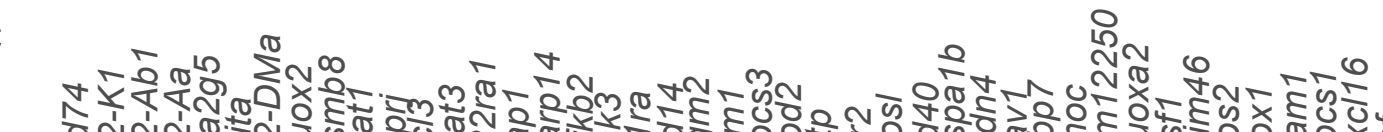

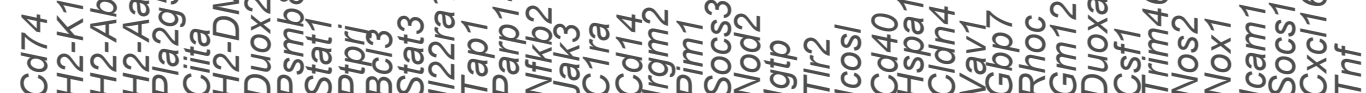

C
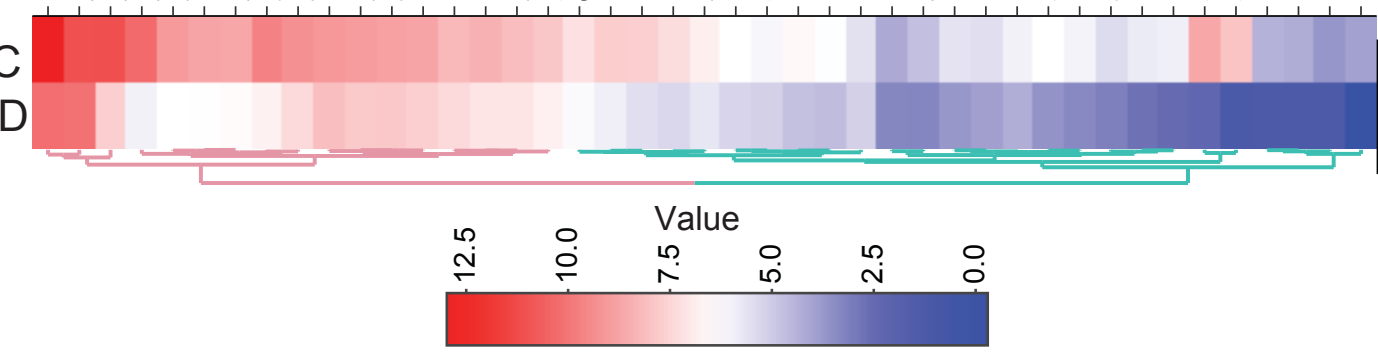

D

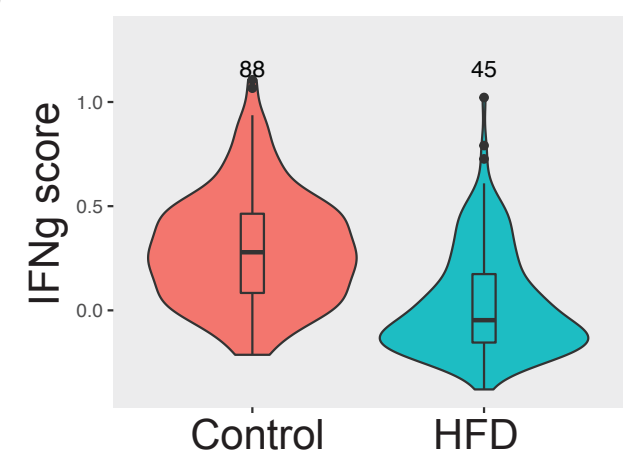

E

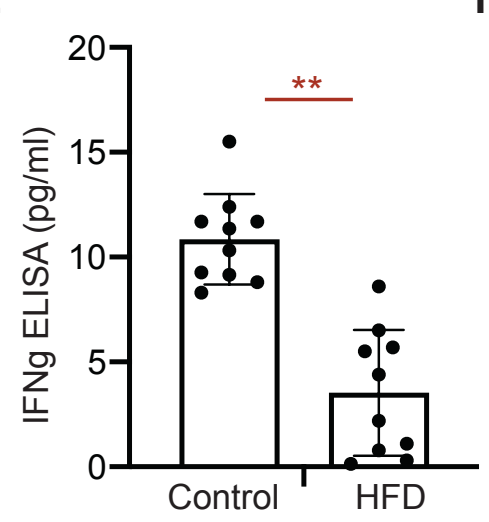

F

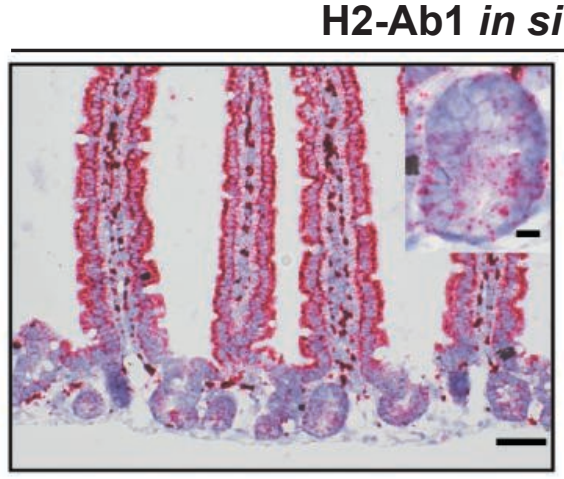

Vehicle tu hybridization
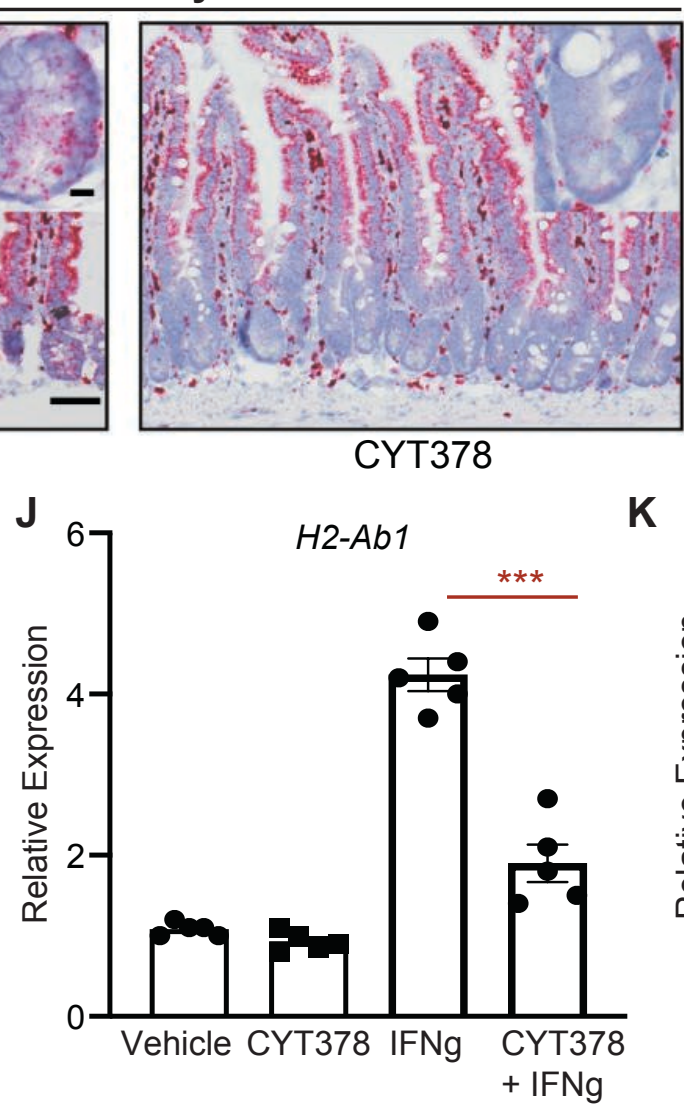

J
G

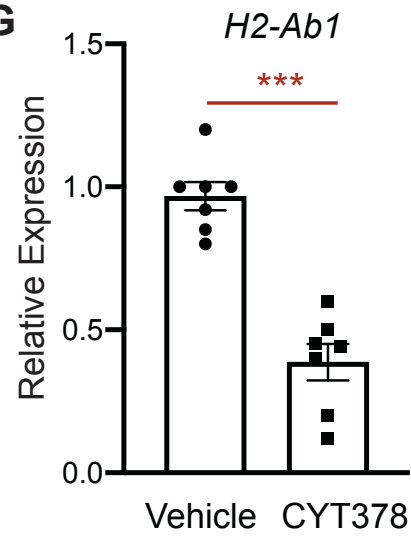

H

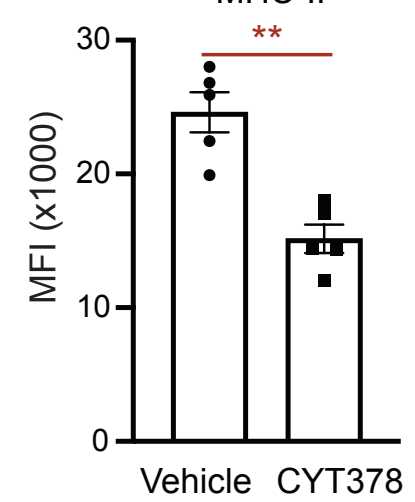

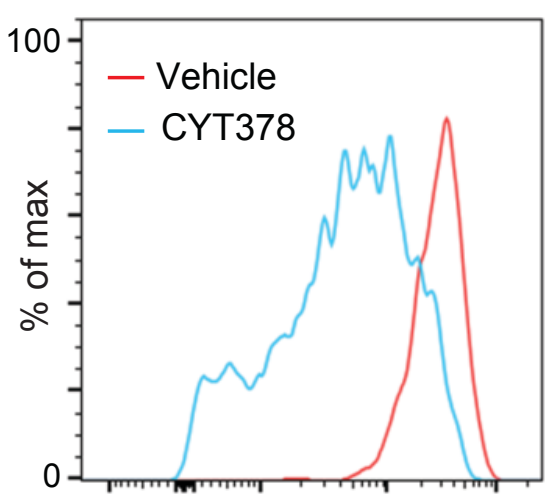

MHC-II PE
K

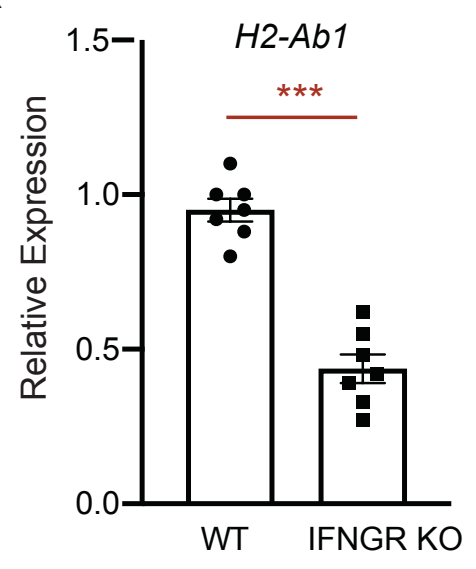


Figure 4

A



D

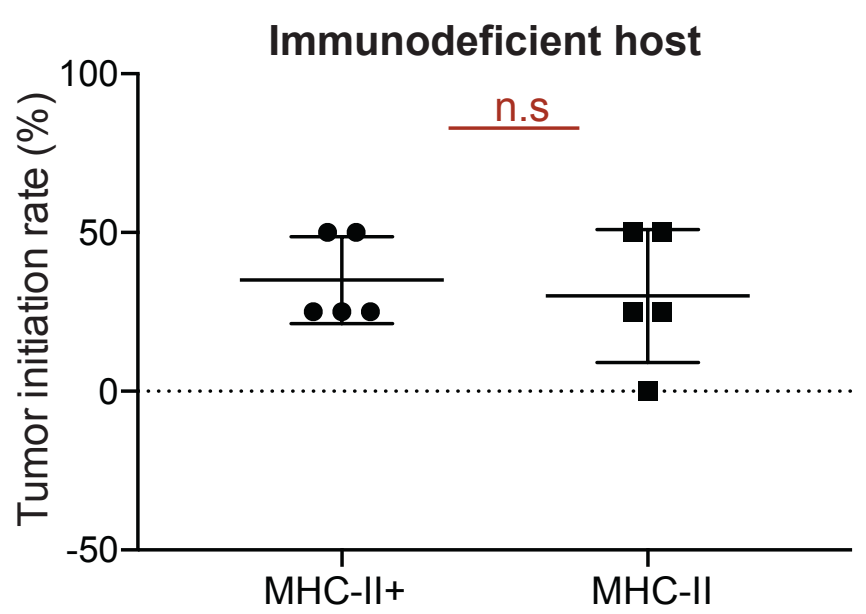

$\mathbf{F}$



B

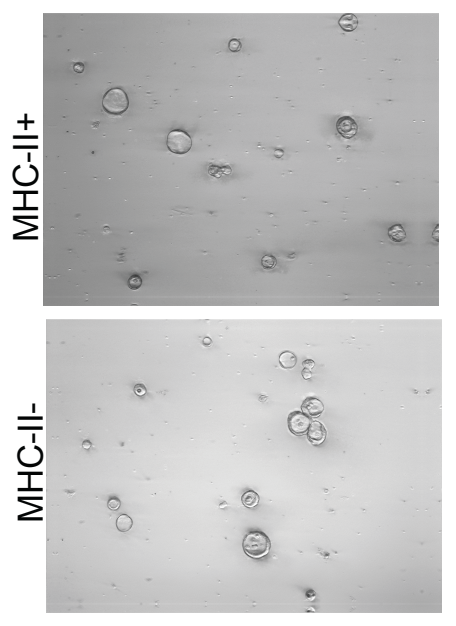

C

Immunocompetent host



E

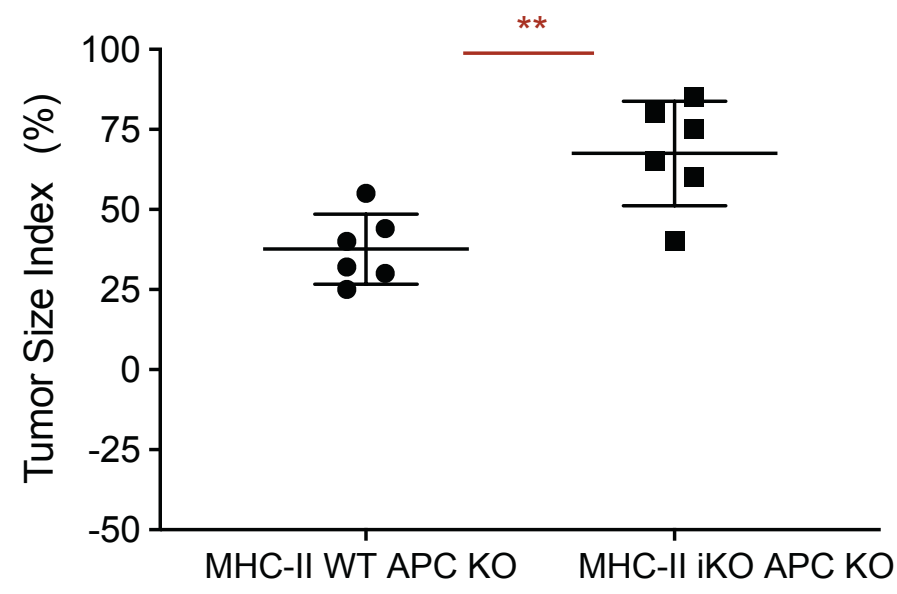

G

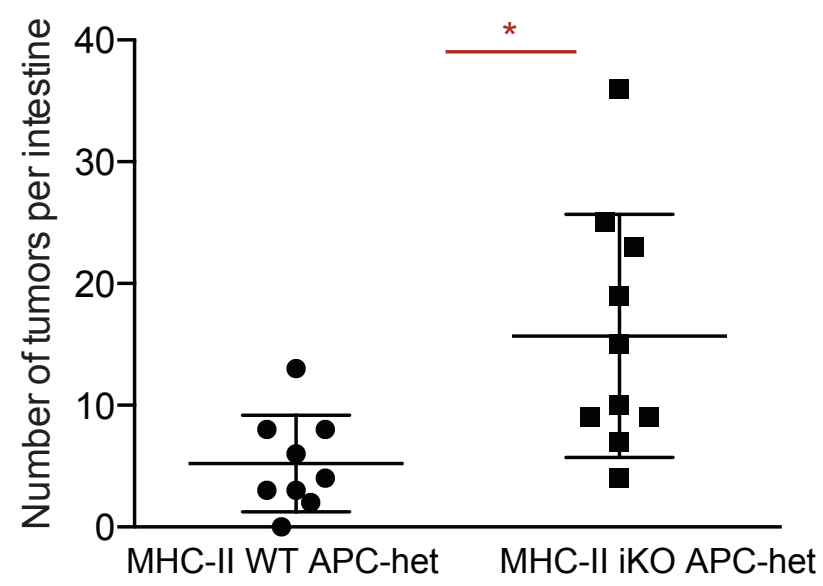


Figure S1

A

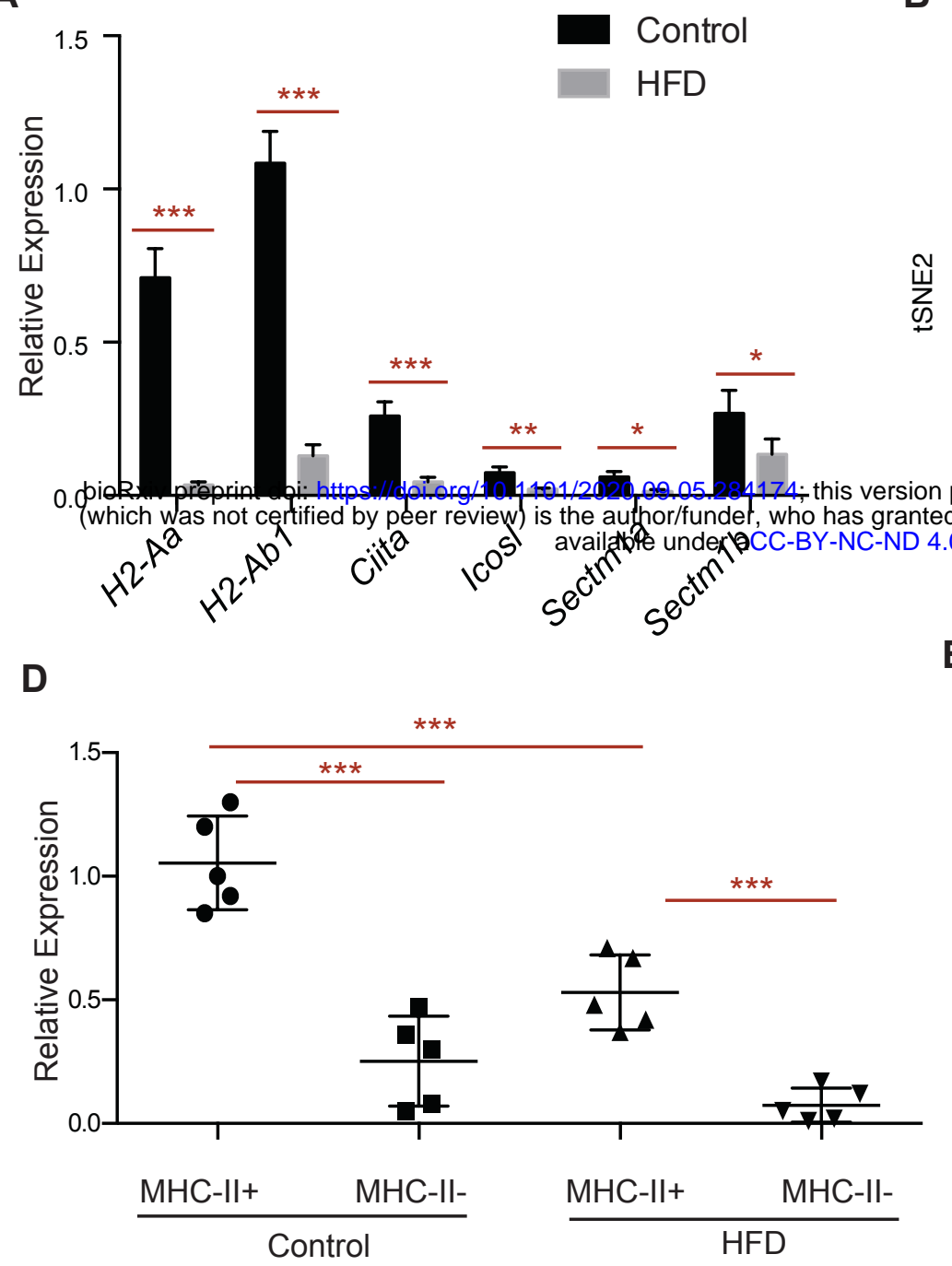

F



G



C

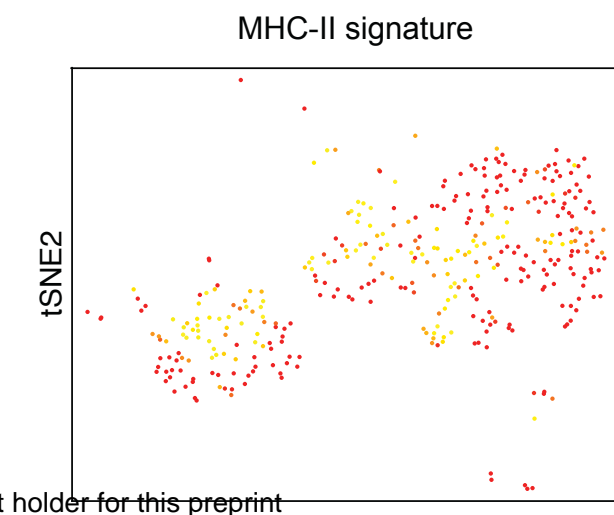

MHC-II signature

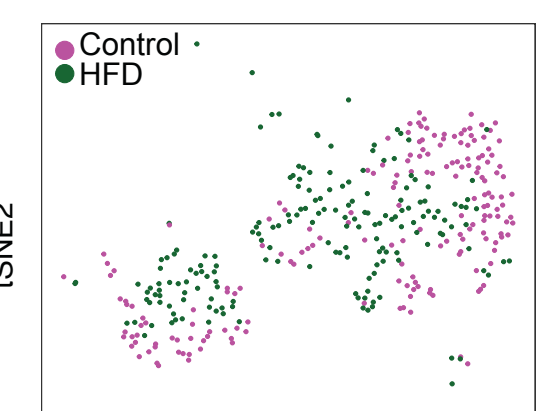

E

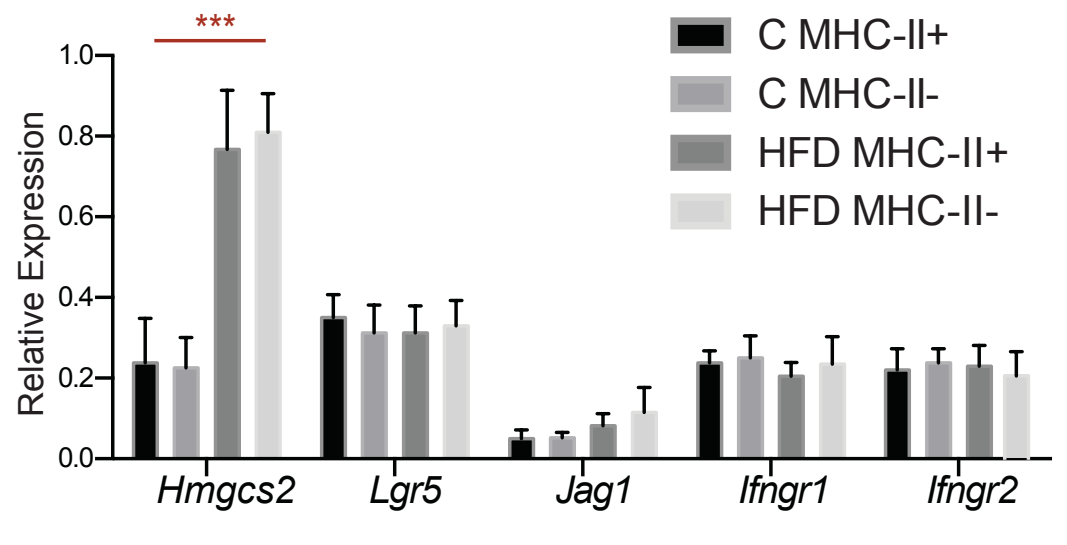

H

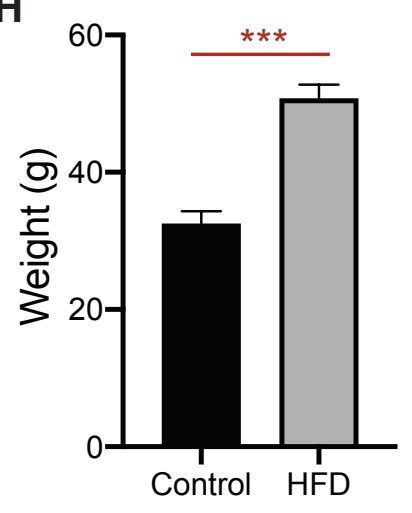

I

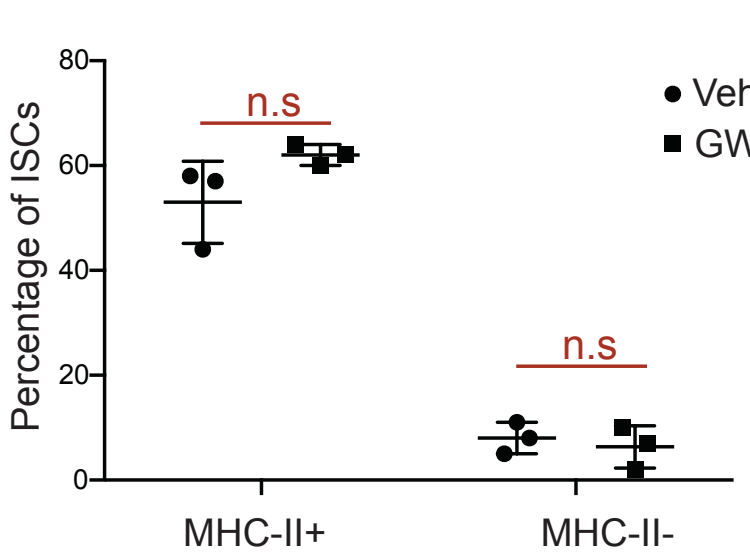

J Vehicle

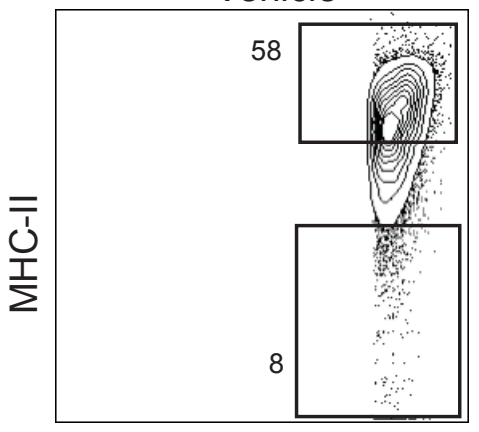

Lgr5-GFP

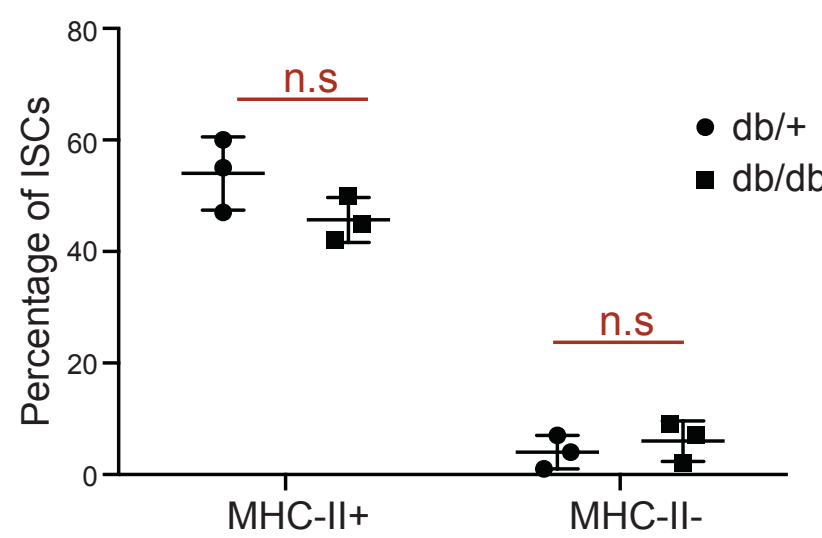

L

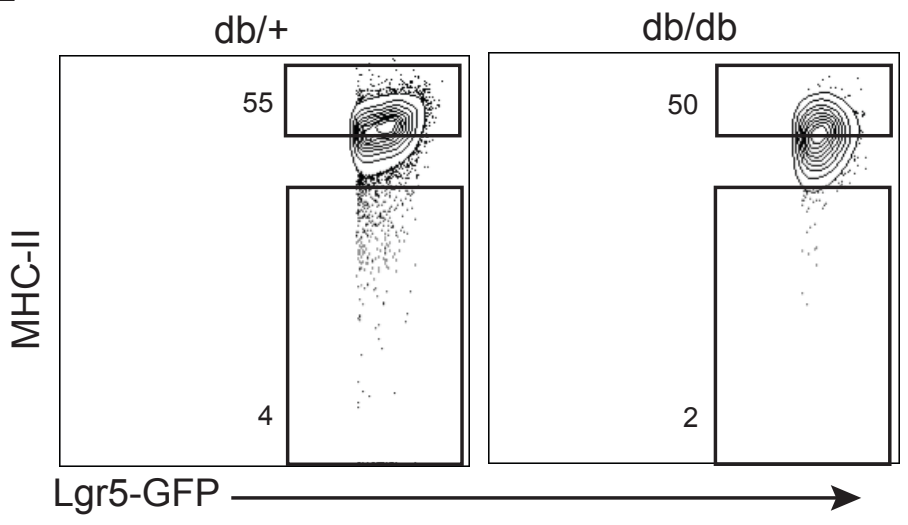


Figure S2

A

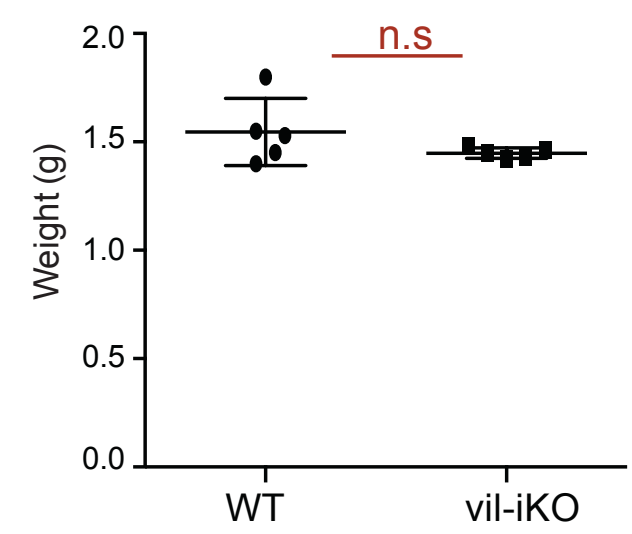

\section{B}

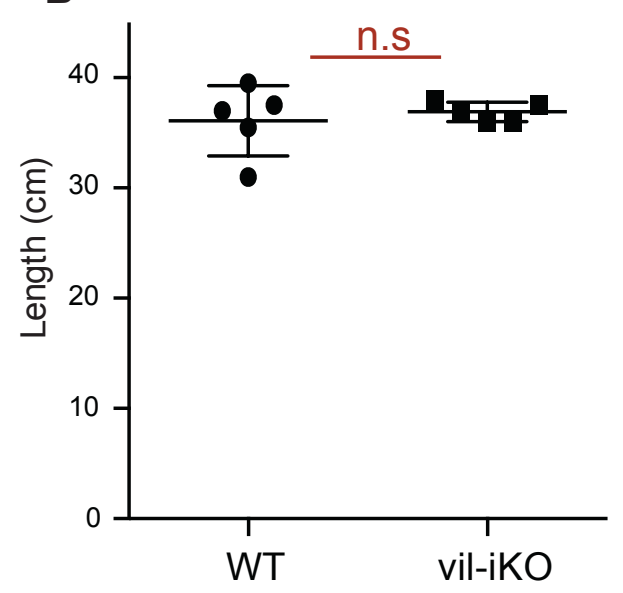

C

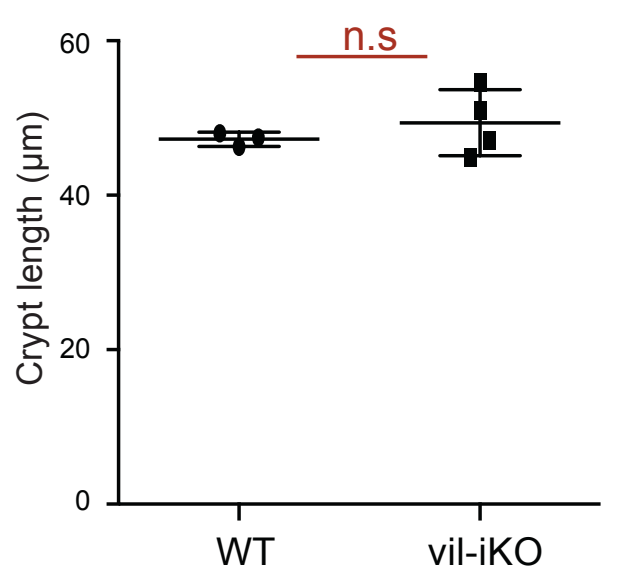

D

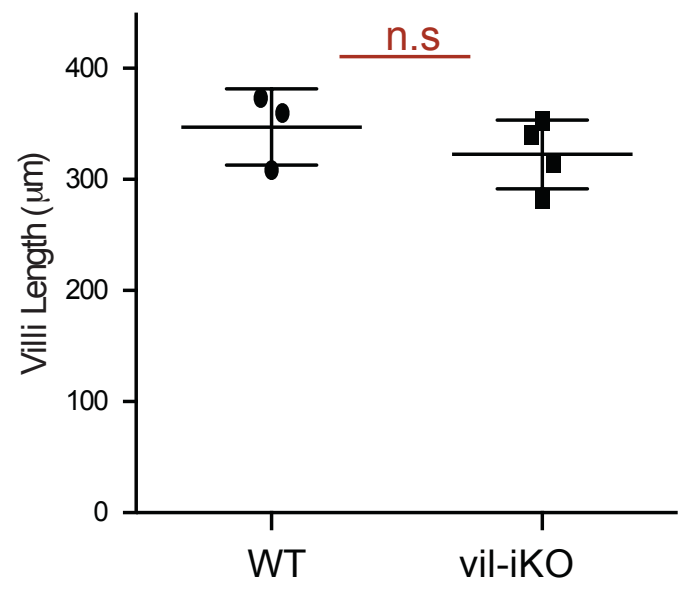

E

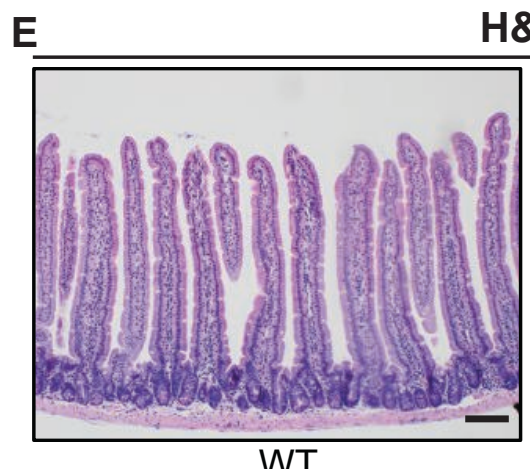

H



Lgr5-GFP
F H2-Ab1 in situ hybridization

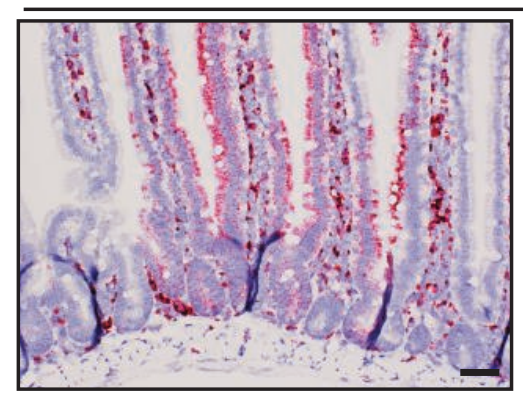

WT

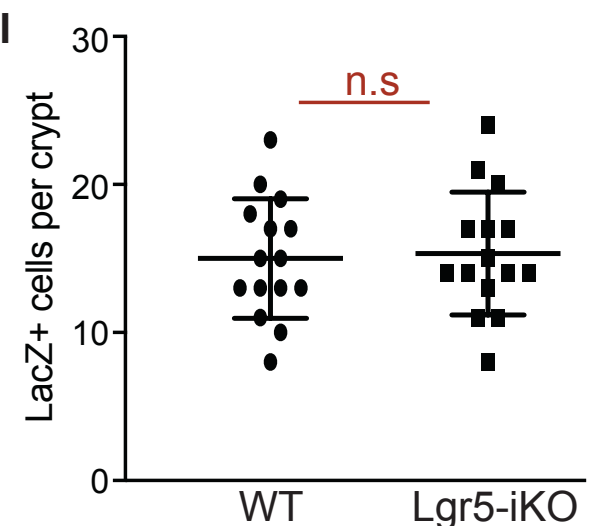

G
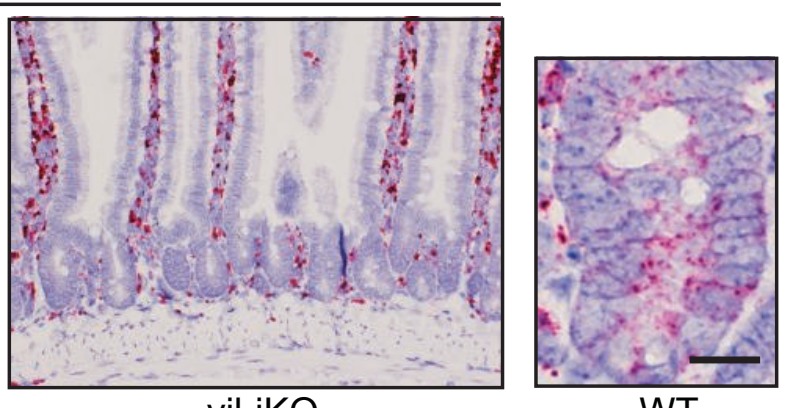

WT

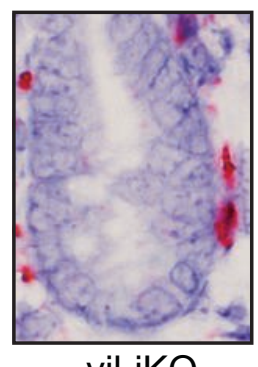

LacZ IHC




Figure S4

A

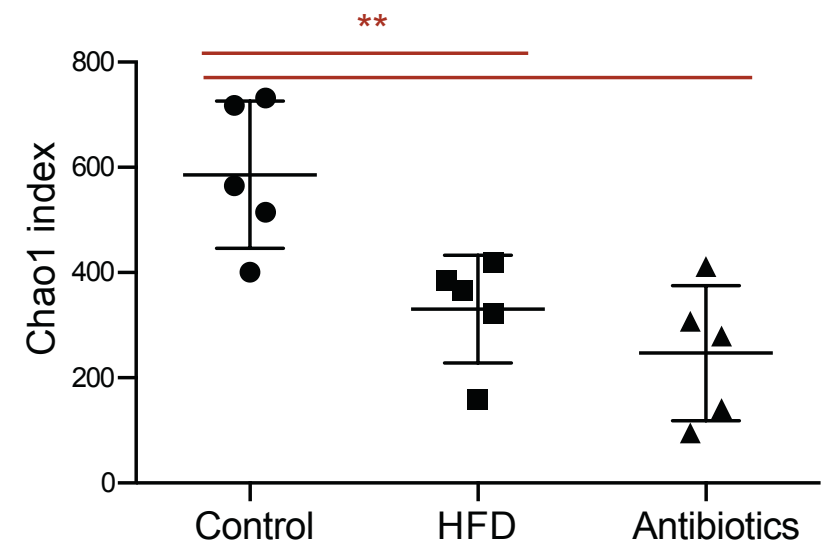

D
$\overline{\bar{U}}$
$\frac{1}{\Sigma}$

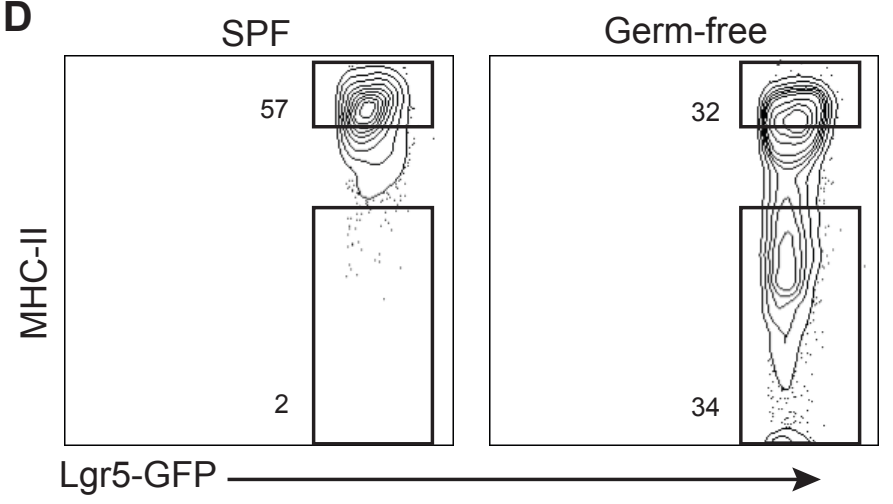

G



E
B
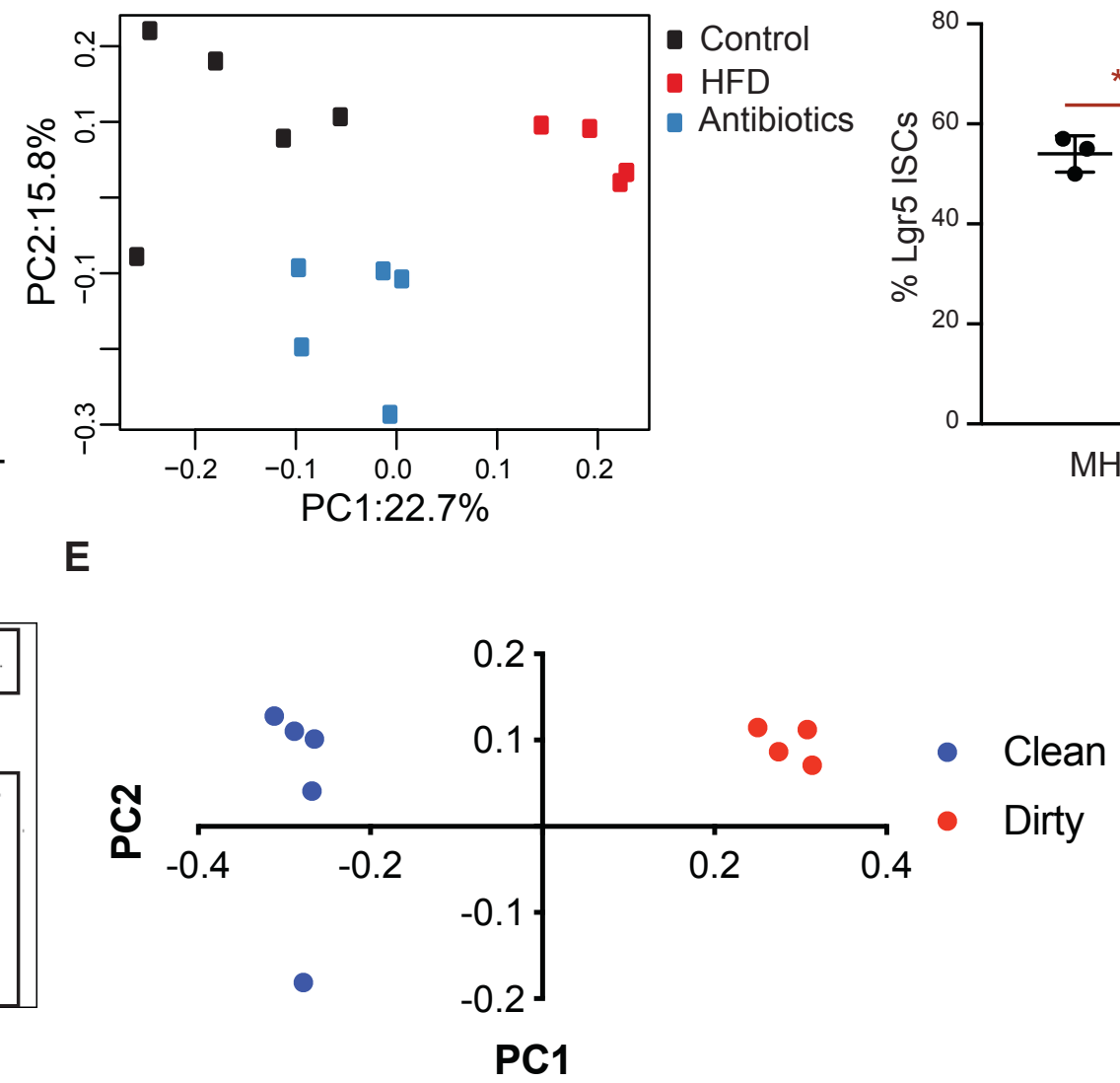

After co-housing

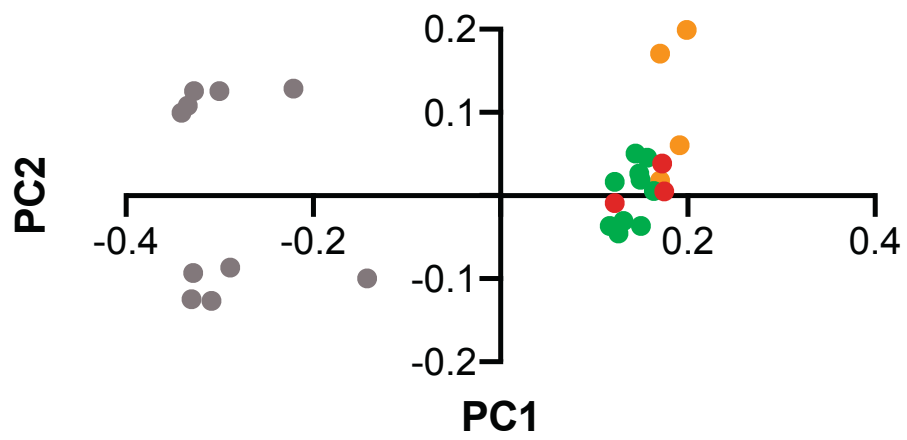

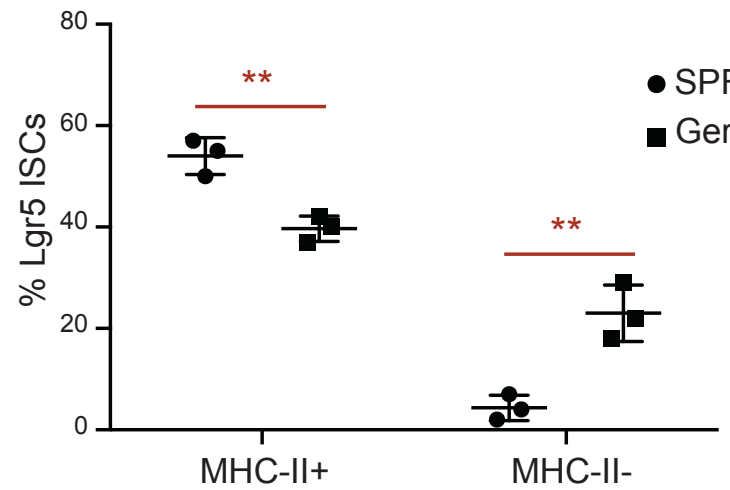

F

Odoribacter sp.



- Clean

- Dirty

- Clean co-housed

- Dirty co-housed 
Figure S5

A

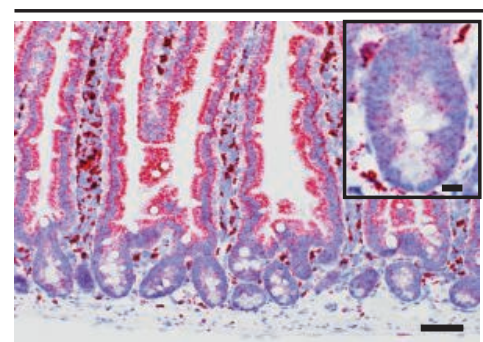

Control + Vehicle

B

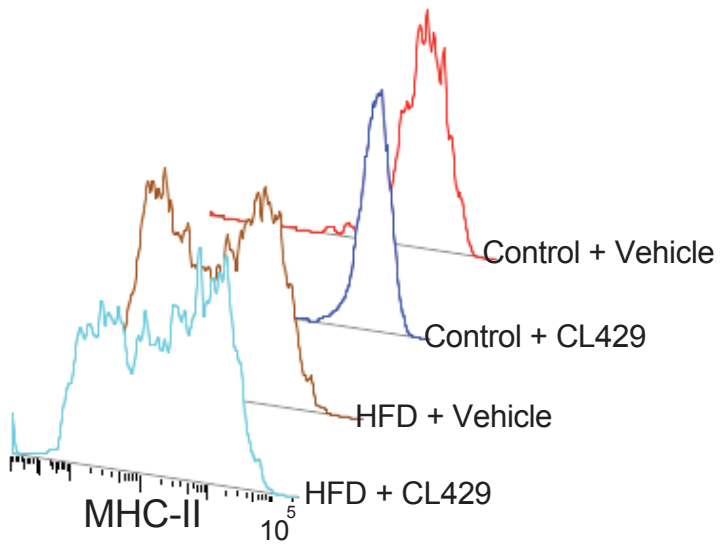

E

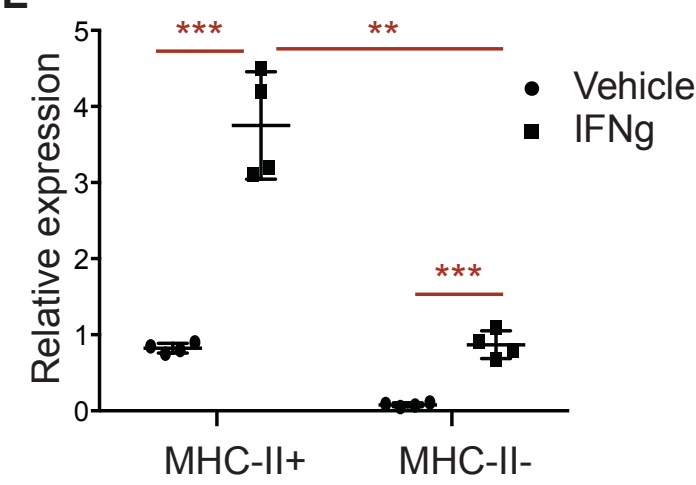

$\mathbf{F}$
H2-Ab1 in situ hybridization

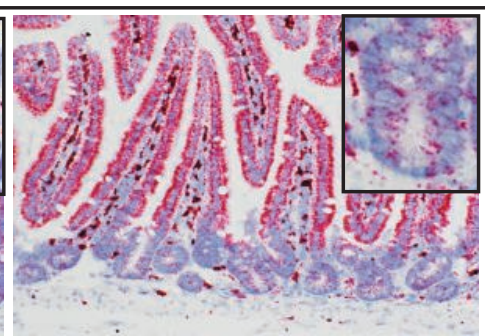

Control + CL429

C
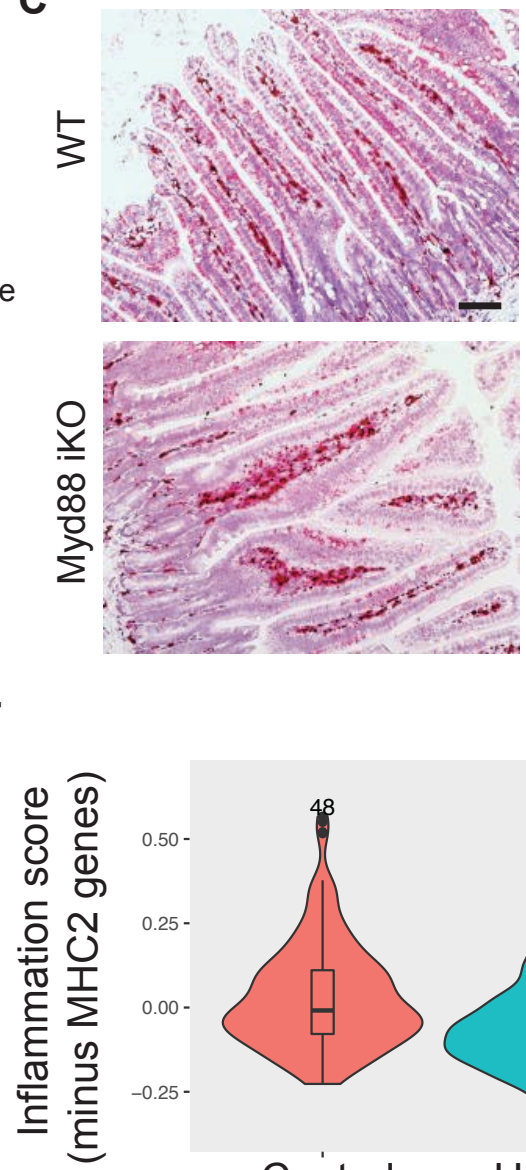

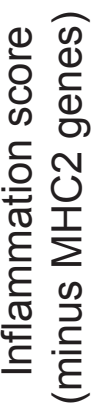

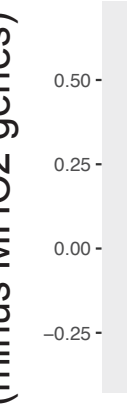

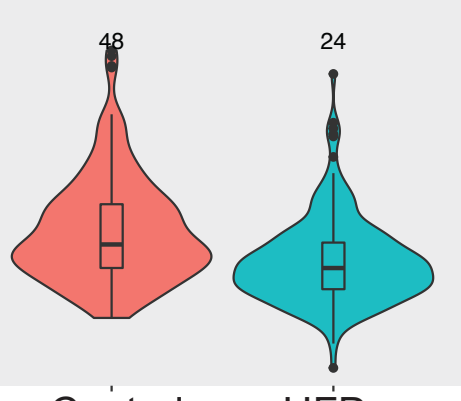

Control HFiD

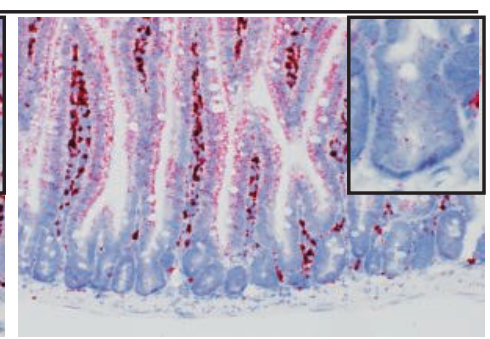

HFD + CL429

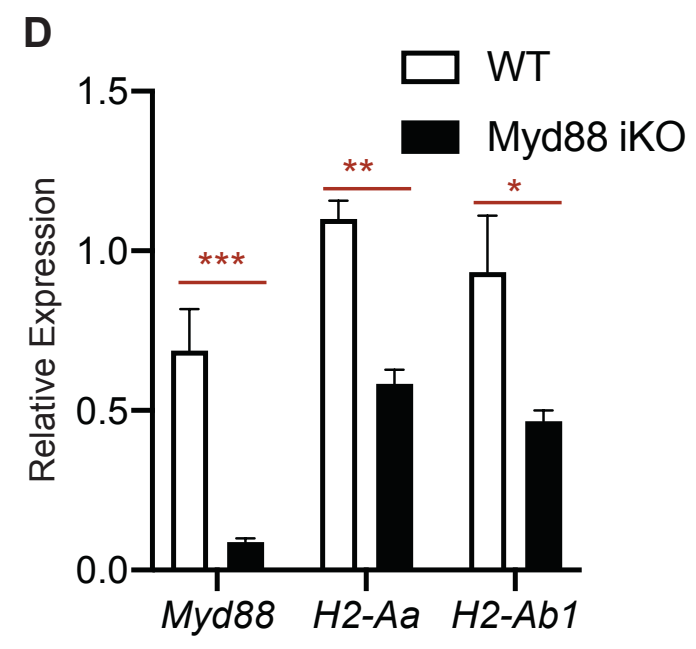

G

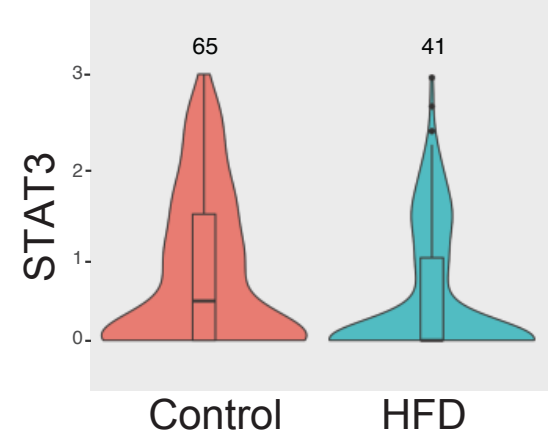

H

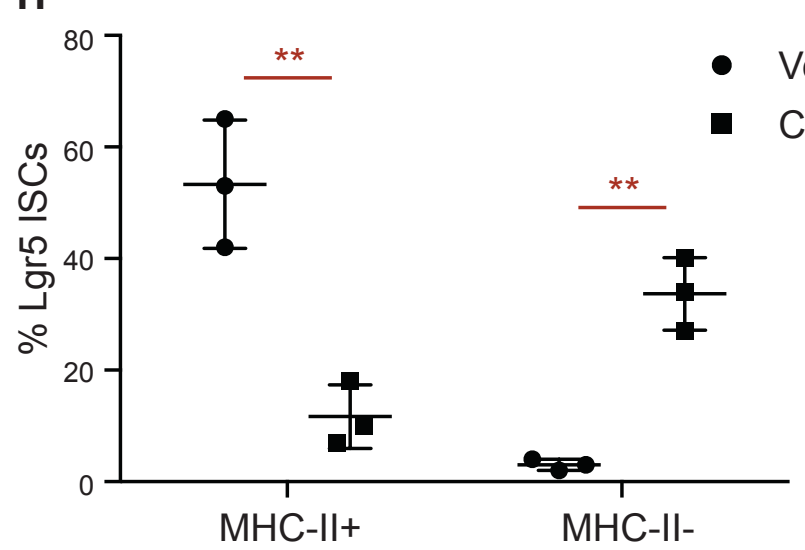

I

Vehicle

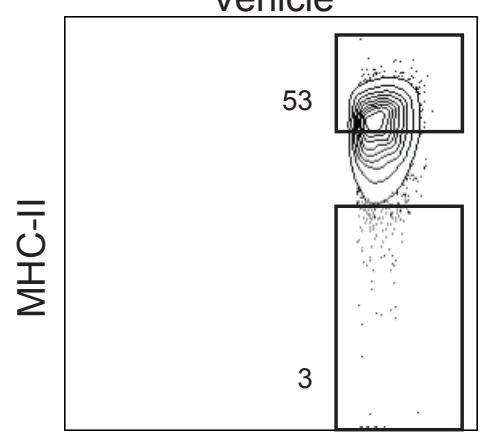

CYT387

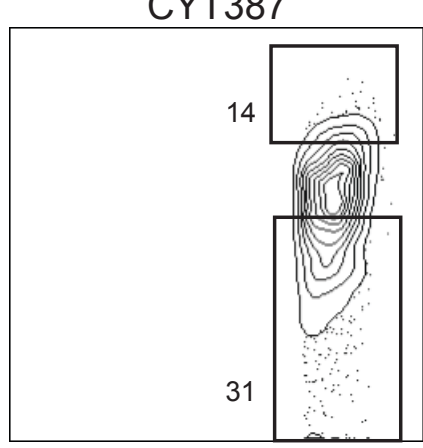

Lgr5-GFP 


\section{Figure S7}

A

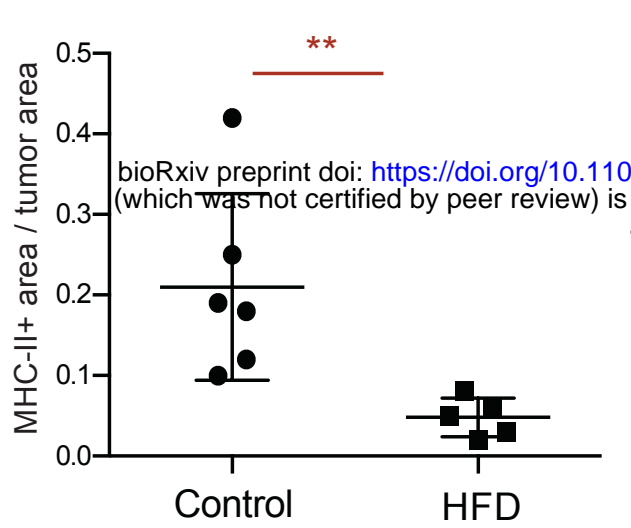

D

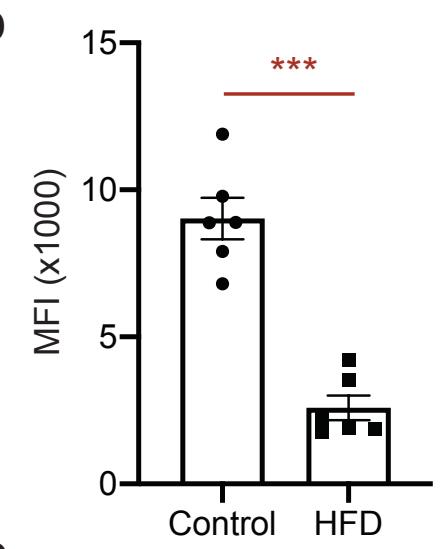

G
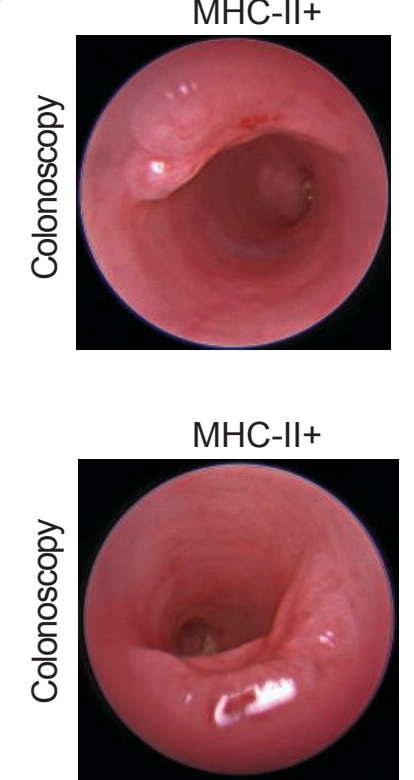

M



$\mathbf{P}$



$\mathbf{Q}$

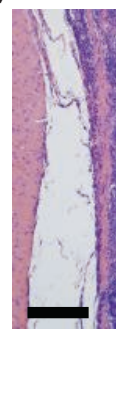

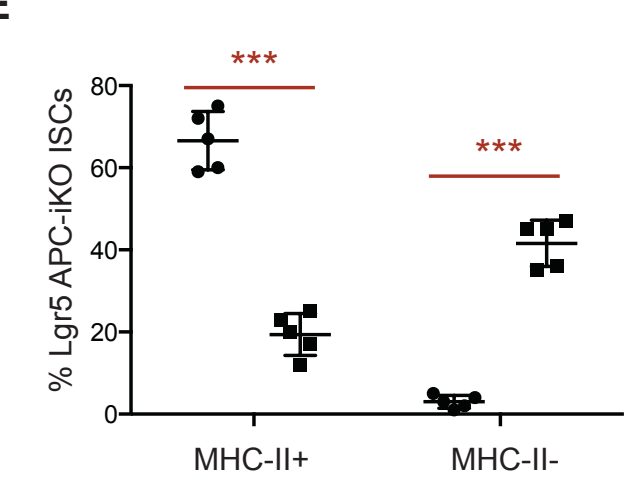

H MHC-II+

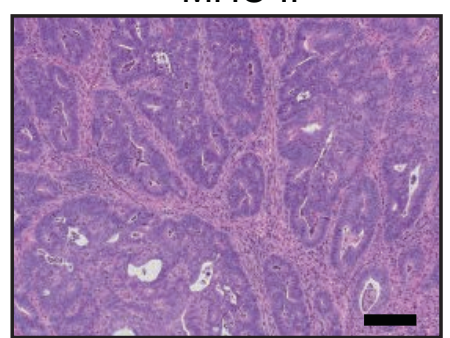

K

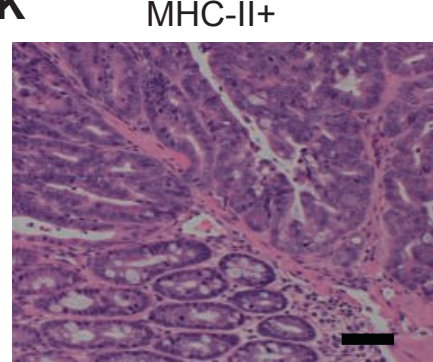

N
HFD

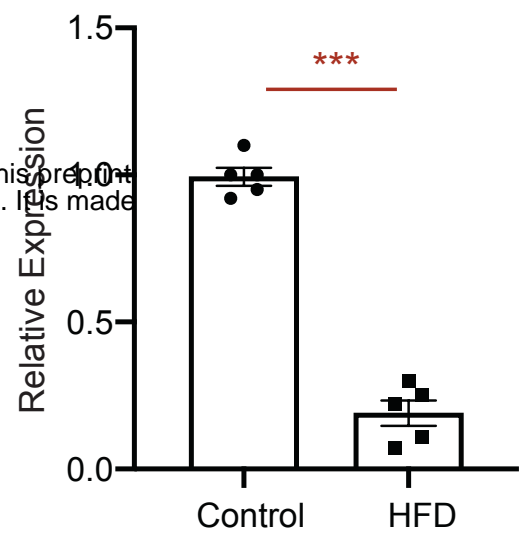

F Control APC-iKO
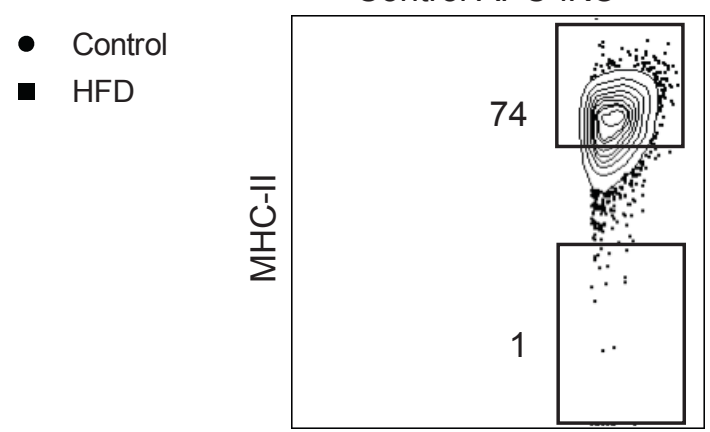

HFD APC-iKO

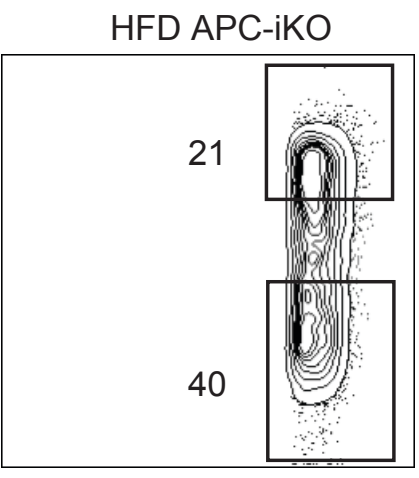

Lgr5-GFP

MHC-II-
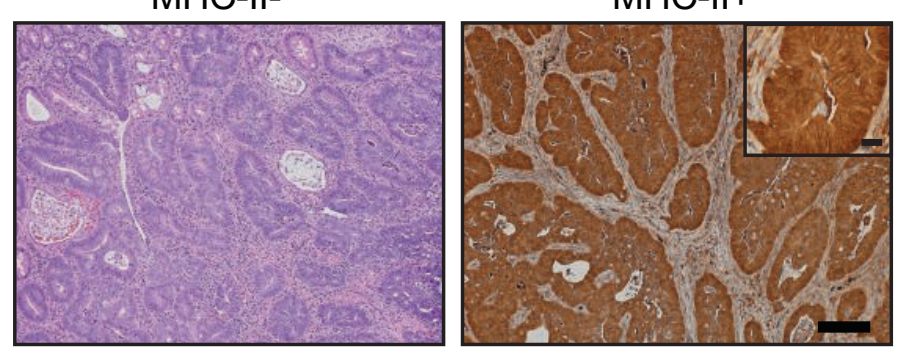

L
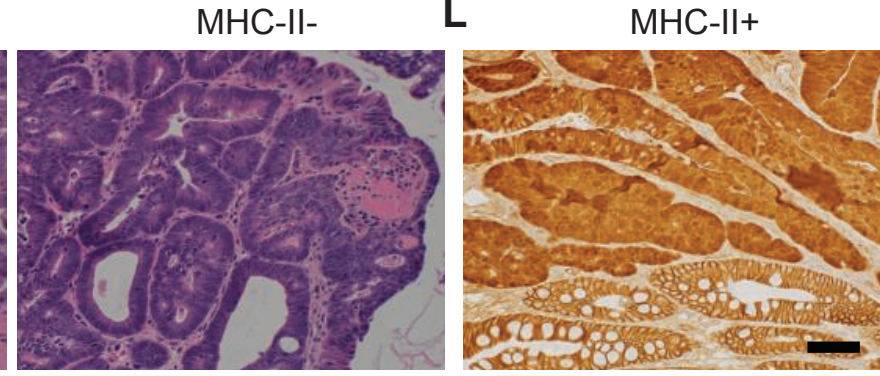

O MHC-II WT

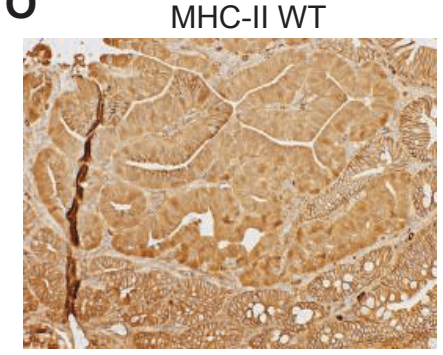

MHC-II L-iKO

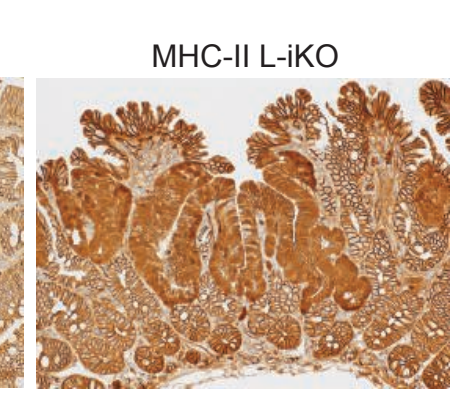

MHC-II WT

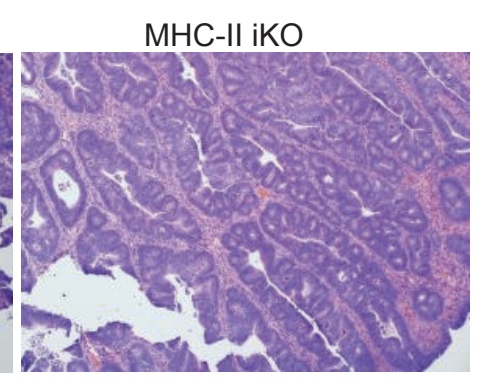

$\mathbf{R}$

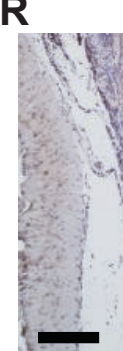

MHC-II WT

MHC-II iKO

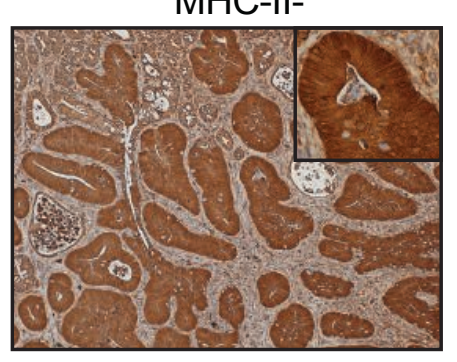

MHC-II-
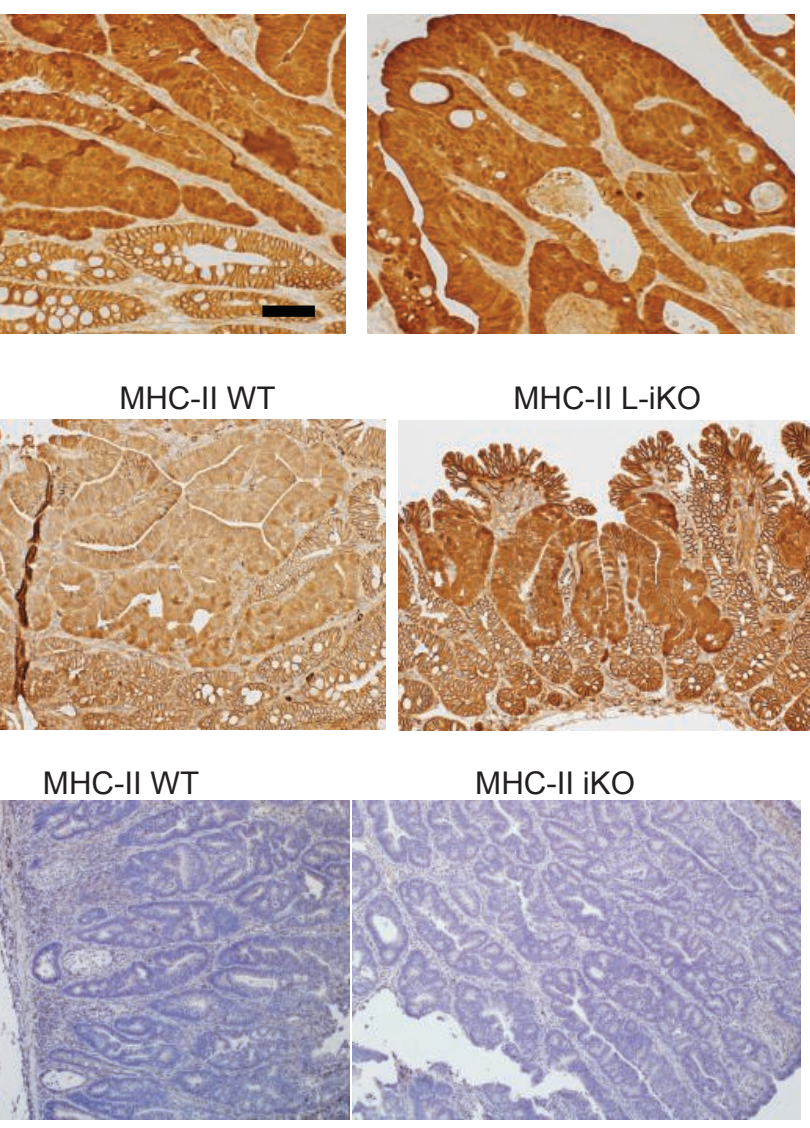